\title{
QGIS: Geografía, Computación, Matemáticas
}

\author{
Vinicio Antonio Gómez Gutiérrez \\ Facultad de Ciencias, Universidad Nacional Autónoma de México \\ e-mail: vgomez@ciencias.unam.mx
}

\begin{abstract}
Resumen
En este artículo se describe a grandes rasgos una aplicación de software libre llamada QGIS, la cual es un sistema de información geográfica. Es muy fácil de aprender, es gratuita, y se puede aplicar tanto en la docencia como en la investigación. Se describen brevemente ejemplos de archivos que contienen información geográfica, algunos de ellos disponibles gracias al Instituto Nacional de Estadística y Geografía. También se da una muestra de las matemáticas que hay detrás, concretamente sobre los sistemas de coordenadas utilizados. Finalmente se comentan algunos estudios donde se han aplicado sistemas de información geográfica en diferentes contextos.
\end{abstract}

\section{Introducción}

Se suele decir que hay que la ciencia y la tecnología están avanzando continuamente, mucho más rápido que los avances en la didáctica y la pedagogía. Muchas veces los maestros que estamos frente a grupo enseñamos lo que aprendimos, como lo aprendimos, sujetos a los tiempos y contenidos que nos marcan los planes y programas de estudio. Considerando que tenemos el desafío de interesar a los jóvenes en el estudio, hagamos la prueba de enseñarles algo nuevo, confiemos en el poder de motivación que tienen la creatividad y la innovación. En este artículo me propongo divulgar una herramienta, llamada QGIS, y cierto tipo de información, que aunque está disponible en la página del Instituto Nacional de Estadística y Geografía (INEGI) desde hace tiempo, en mi opinión, no está suficientemente utilizada en las universidades e instituciones de educación superior y media superior.

El sistema QGIS, (Quantum Geographical Information System), es un software libre y de código abierto que nos proporciona una aplicación GIS para el manejo y análisis de información geográfica que podemos desplegar en forma de mapas. Con QGIS podemos abrir archivos que tienen mapas digitalizados. Podemos consultar la información que está codificada en dichos mapas, y producir nuevos mapas en los cuales se resalte el resultado de nuestra consulta. Esto permite visualizar relaciones espaciales que no podríamos apreciar en una consulta de una base de datos ordinaria. También podemos combinar la información de dos o más mapas distintos, e integrarla para producir un nuevo mapa en el cual podamos estudiar las relaciones espaciales entre las variables que estén representadas en los mapas que tomaríamos como insumo. La página web del proyecto QGIS es

$$
\text { http://qgis.org/en/site/ }
$$


De este sitio es posible descargar el citado software para instalarlo en nuestras computadoras personales, así como la documentación necesaria para aprender a usarlo. El proyecto tiene su origen en 2002 y ha tenido una rápida expansión desde entonces por su facilidad de manejo y sus múltiples aplicaciones, además, el hecho de que sea gratuito facilita el acceso al mismo por parte de estudiantes e instituciones de educación superior.

En la primera sección se describen algunos ejemplos de archivos con información geográfica elaborados por el INEGI. Uno de ellos es la carta topográfica correspondiente a Cananea, el otro de ellos es la parte del continuo de elevaciones mexicano correspondiente a Sonora. Se describe cómo utilizar QGIS para leer estos archivos y consultar la información contenida en ellos.

En la segunda sección se describen dos maneras de representar información geográfica en la computadora; concretamente se describe cómo utilizar QGIS para producir archivos con extensión .shp y los archivos con extensión .xyz, los cuales son ejemplos de conjuntos de datos vectoriales (shape files) y conjunto de datos en formato de malla rectangular (grid o ráster).

En la tercera sección se describen a grandes rasgos los sistemas de coordenadas utilizados en cada uno de los archivos que se tomaron como ejemplo. Uno de estos sistemas de coordenadas es el de latitud-longitud, pero cabe resaltar que en él se modela a la superficie terrestre más como un elipsoide que como una esfera. El otro de los sistemas coordenados utilizados es el sistema de coordenadas Universal Transversal de Mercator (UTM).

En la última sección se mencionan ejemplos de aplicaciones más avanzadas, en diferentes contextos, muchos de ellos muy interesantes.

\section{Consultando archivos del INEGI}

\subsection{Cartas Topográficas}

En la página del INEGI [12] se pueden descargar cartas topográficas que representan diferentes regiones de nuestro país, a diferentes escalas. Una de ellas es la carta topográfica H12B53 (Cananea) escala 1:50 000, en formato de datos vectoriales.

Ejemplo de consulta de datos (16 de junio de 2016)

En la página www.inegi.org.mx seleccioné Geografía, Temas, Topografía. Accedí a la página www.inegi.org.mx/geo/contenidos/topografia/default.aspx. En Productos y servicios seleccioné Cartas topográficas. 


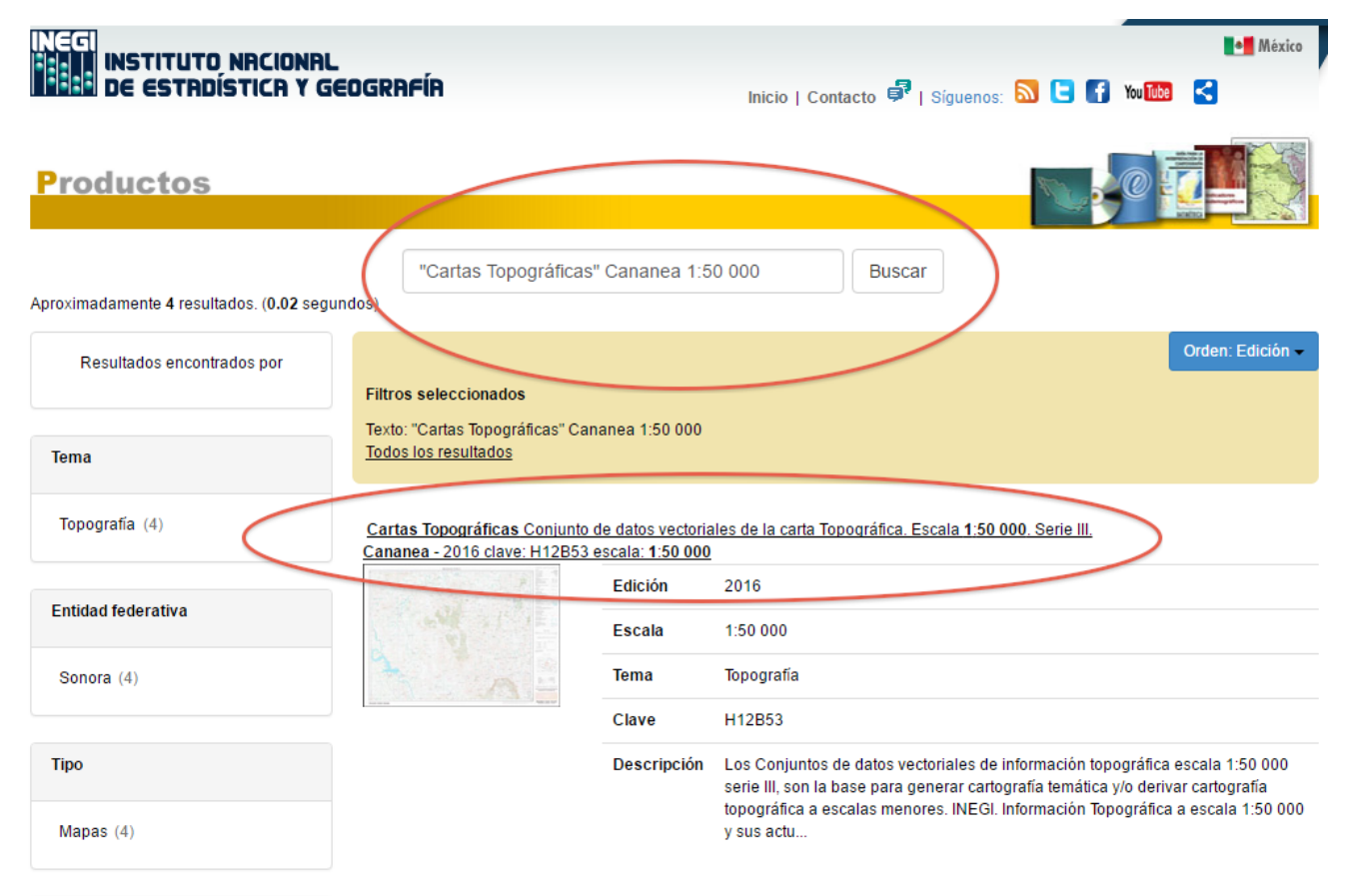

Figura 1: Búsqueda de una carta topográfica

Aparecen cinco versiones de la carta topográfica que buscamos.

La más reciente es de 2016.

Supongamos que ya la descargamos y desempaquetamos en nuestra computadora. Dicha carta está conformada por un conjunto de archivos con diferentes nombres y extensiones. Supongamos que también tenemos instalada una versión de QGIS compatible con nuestro sistema operativo (se puede descargar desde la página de QGIS [16]). Para abrir uno de los archivos de la carta topográfica se procede como sigue:

Abramos la aplicación QGIS. Aparecerá una imagen como la siguiente (dependiendo de la versión de QGIS que se instale).

Seleccione Capa, Añadir capa, Añadir capa vectorial

Aparece un cuadro de diálogo. Seleccione Explorar

Vaya al directorio en el cual desempaquetó el archivo zip. Seleccione el archivo de curvas de nivel con extensión shp (Dato auxiliar: tiene 7,423 KB).

\section{Seleccione Open}

Seleccione Abrir

Aparece un cuadro de diálogo para especificar el Sistema de Referencia de Coordenadas (SRC). 


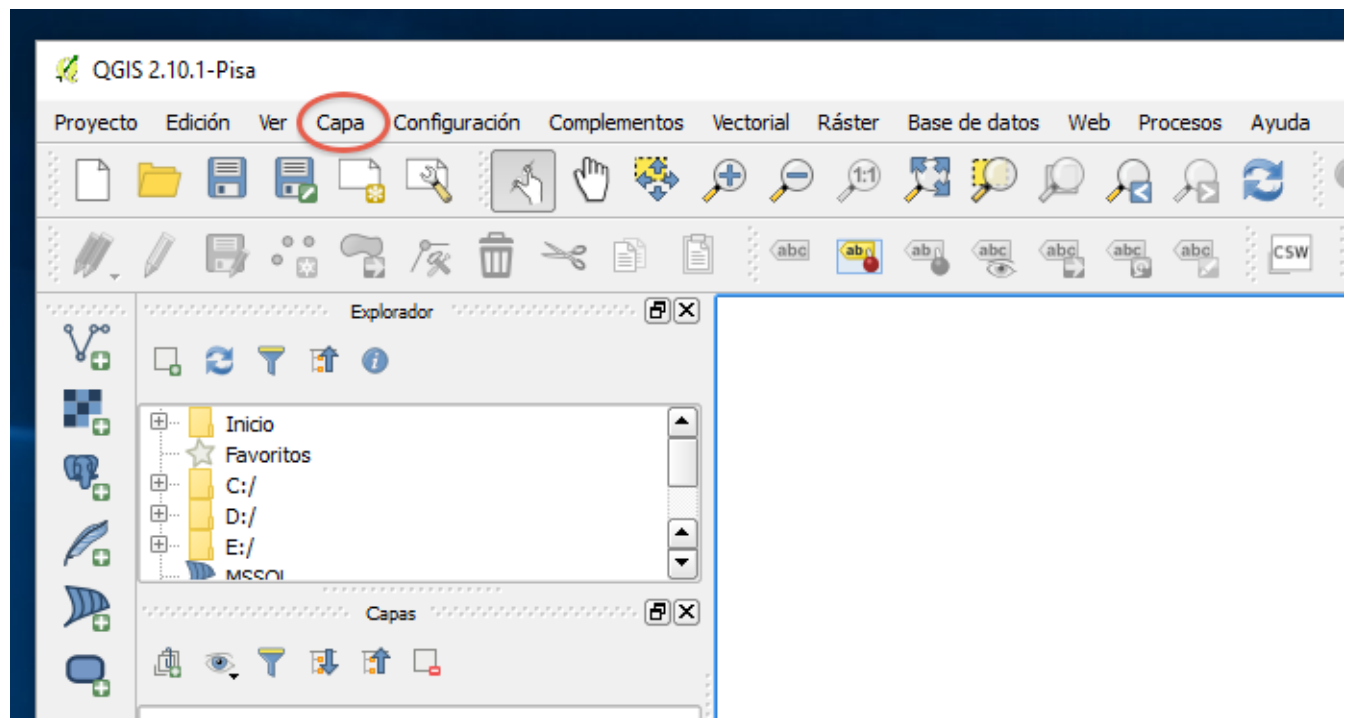

Figura 2: Añadir una capa

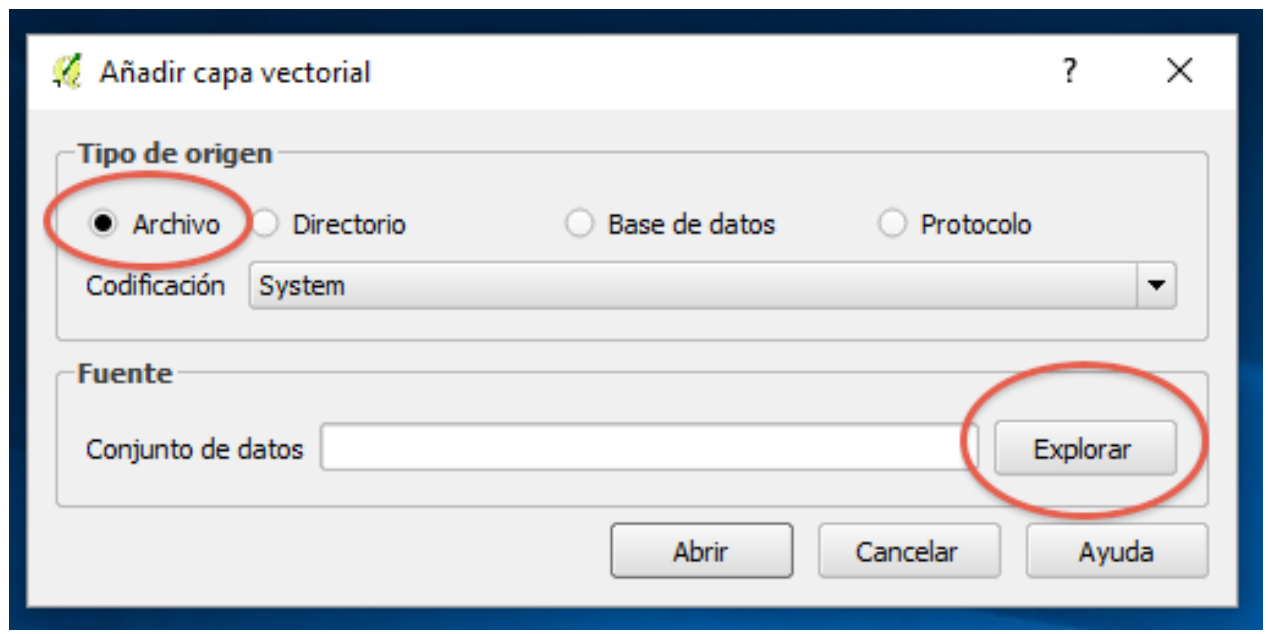

Figura 3: Explore

Seleccione el Sistema de Referencia de Coordenadas WGS 84 / UTM zone 12N con código EPSG:3612. Una vez hecho esto, seleccione Aceptar

QGIS nos desplegará el mapa con la capa de curvas de nivel.

Se puede modificar la apariencia de la capa como sigue:

En la pestaña Capa seleccione Propiedades

Aparecerá un cuadro de diálogo

En la opción de Estilo modifique la opción de representar por Símbolo único

Seleccione Graduado

En la opción Rampa de color seleccione RdYIGn

Oprima los botones Clasificar, Aplicar, Aceptar 


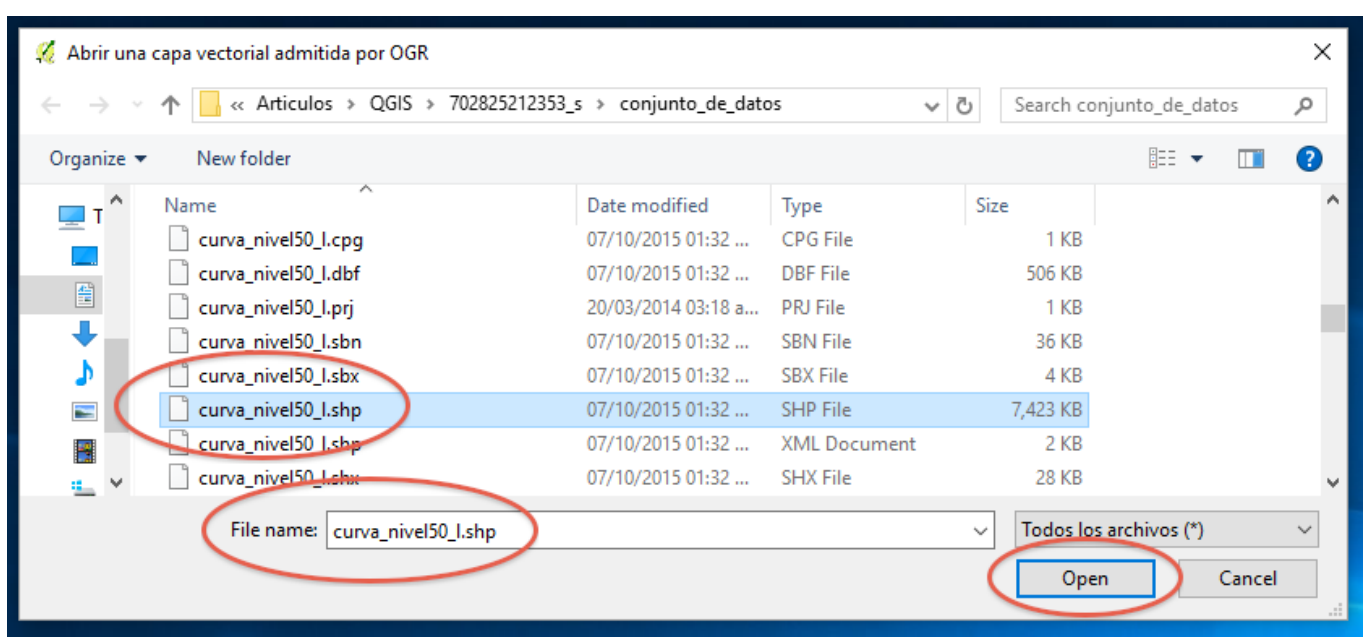

Figura 4: Seleccione un archivo tipo shp

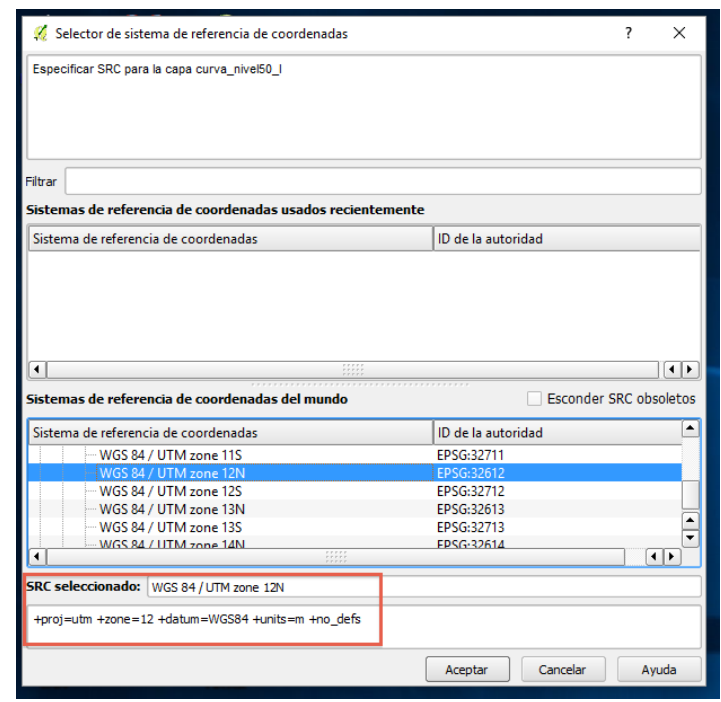

Figura 5: Se debe elegir el sistema de coordenadas en el que se elaboró el archivo que contiene los datos

Para mostrar otra funcionalidad de QGIS, podemos añadir la capa de localidades representadas por polígonos (el archivo llamado localidad 50 a.shp).

Para que QGIS despliegue el nombre de la localidad se procede como sigue:

Se abre el menú Propiedades.

Se elige Etiquetas

Se marca el cuadrito Etiquetar esta capa

Se selecciona el campo NOMBRE

Click en Aplicar, Aceptar 


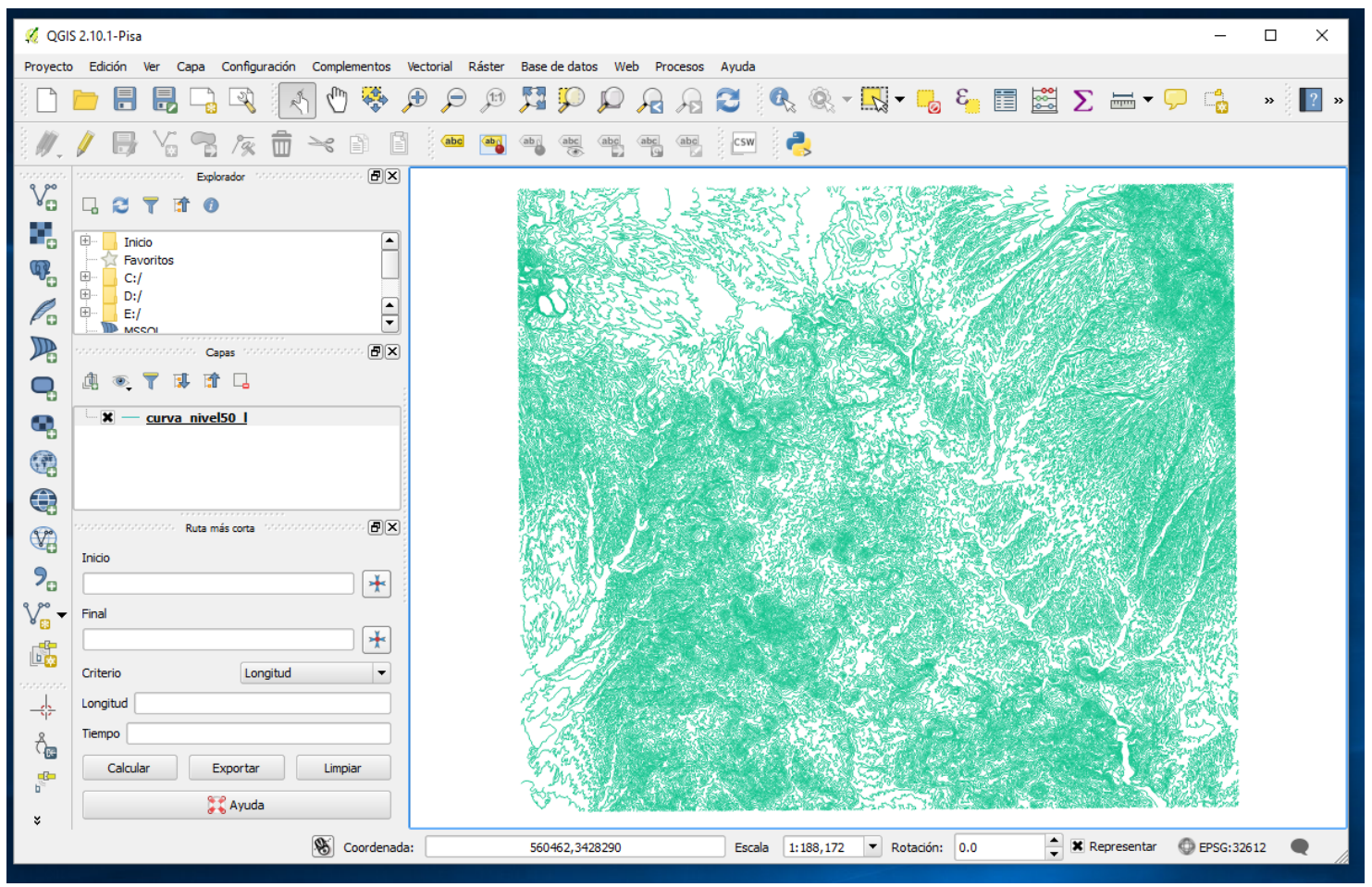

Figura 6: Curvas de nivel de la carta H12B53 Cananea

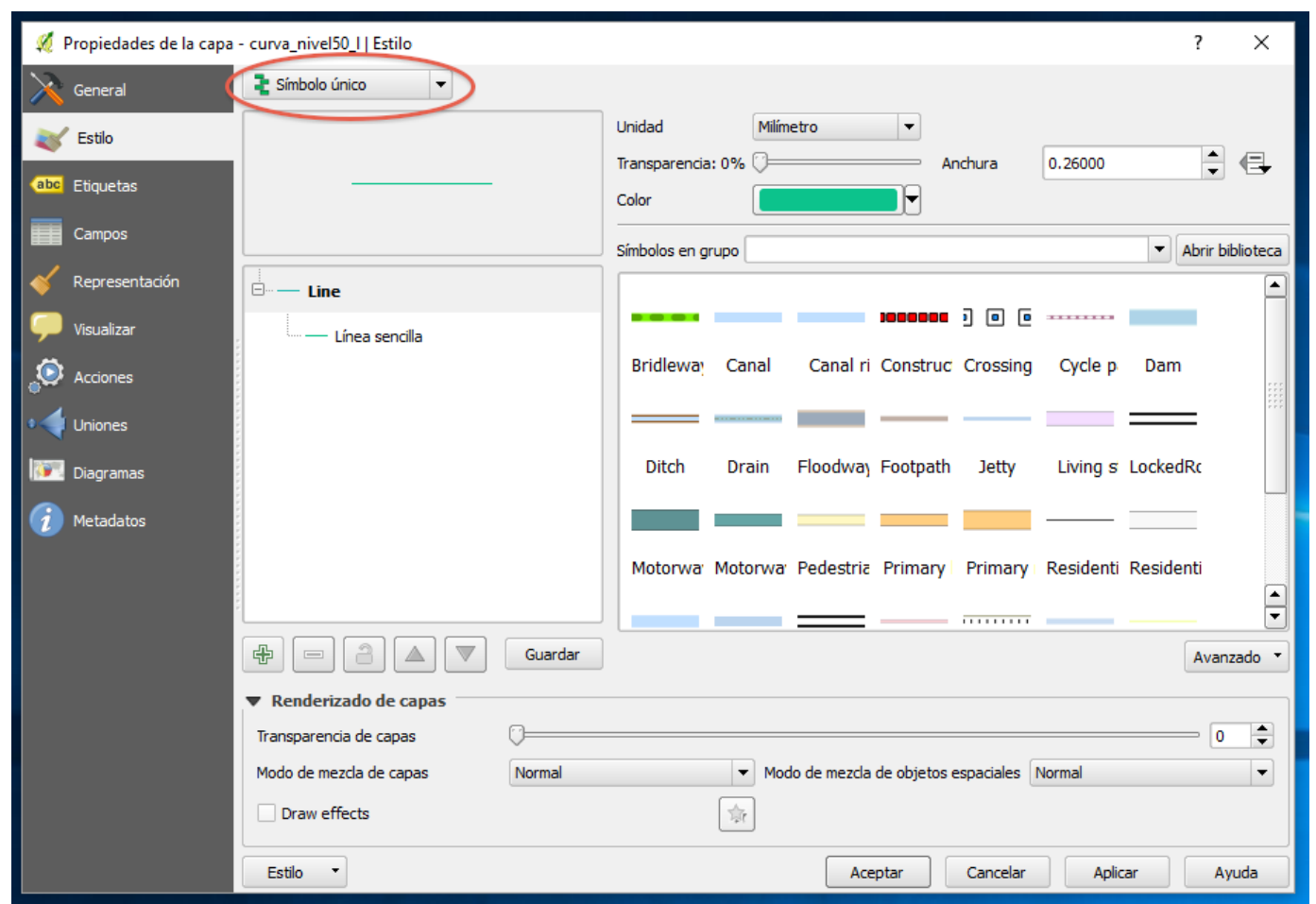

Figura 7: Cuadro de diálogo de propiedades de la capa de curvas de nivel 


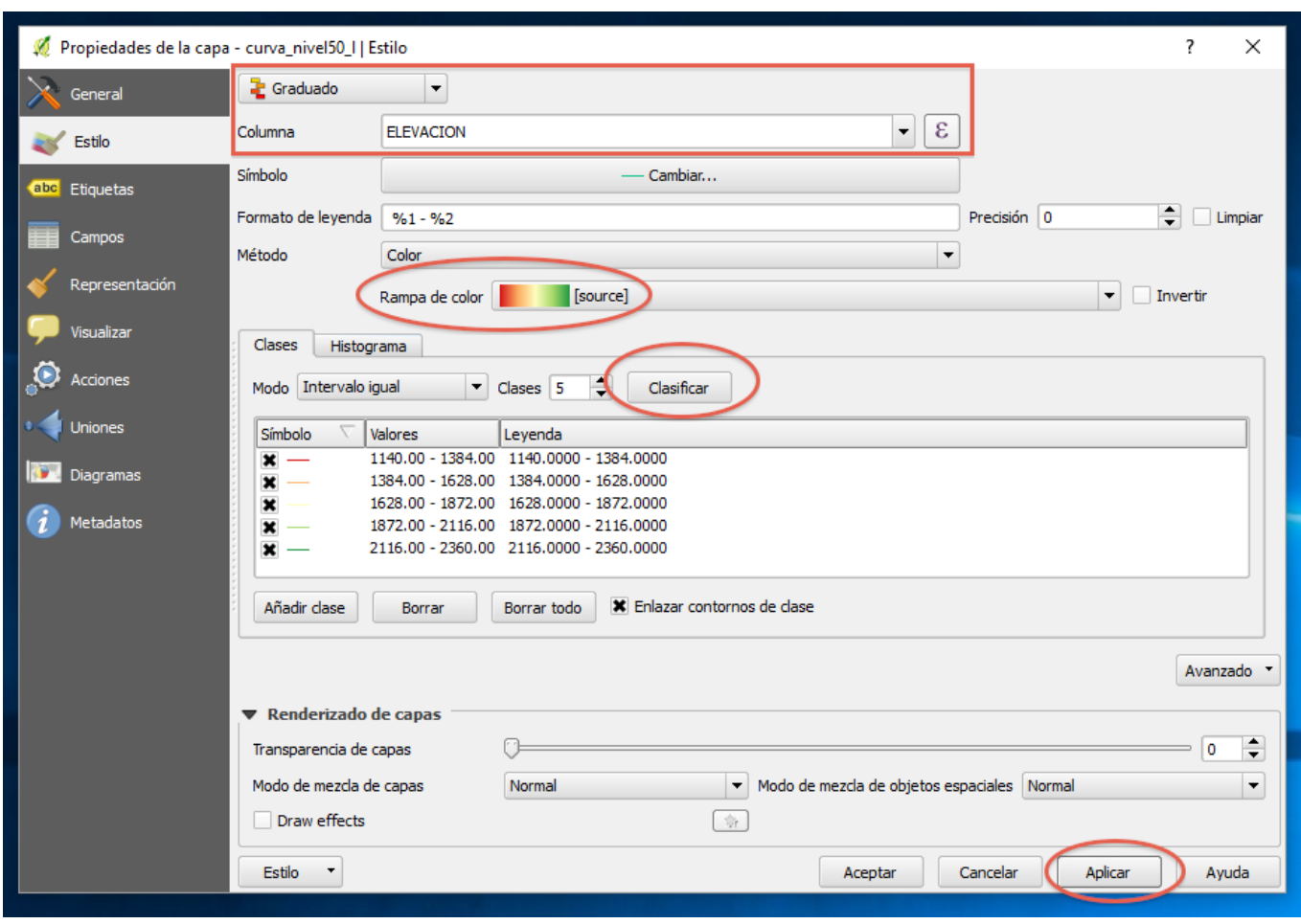

Figura 8: Modificando la manera de visualizar la capa de curvas de nivel

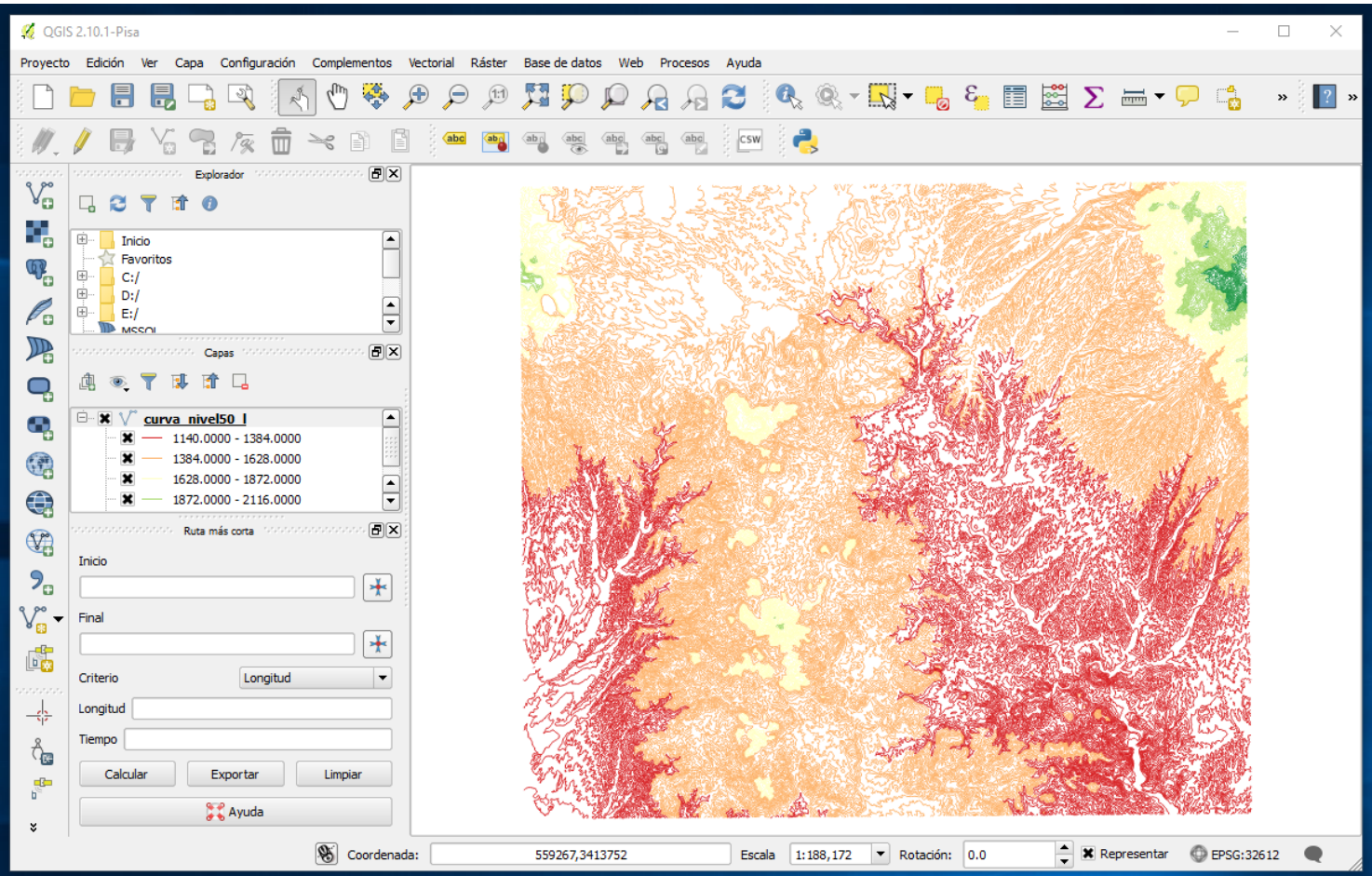

Figura 9: Capa de curvas de nivel coloreando según la elevación 


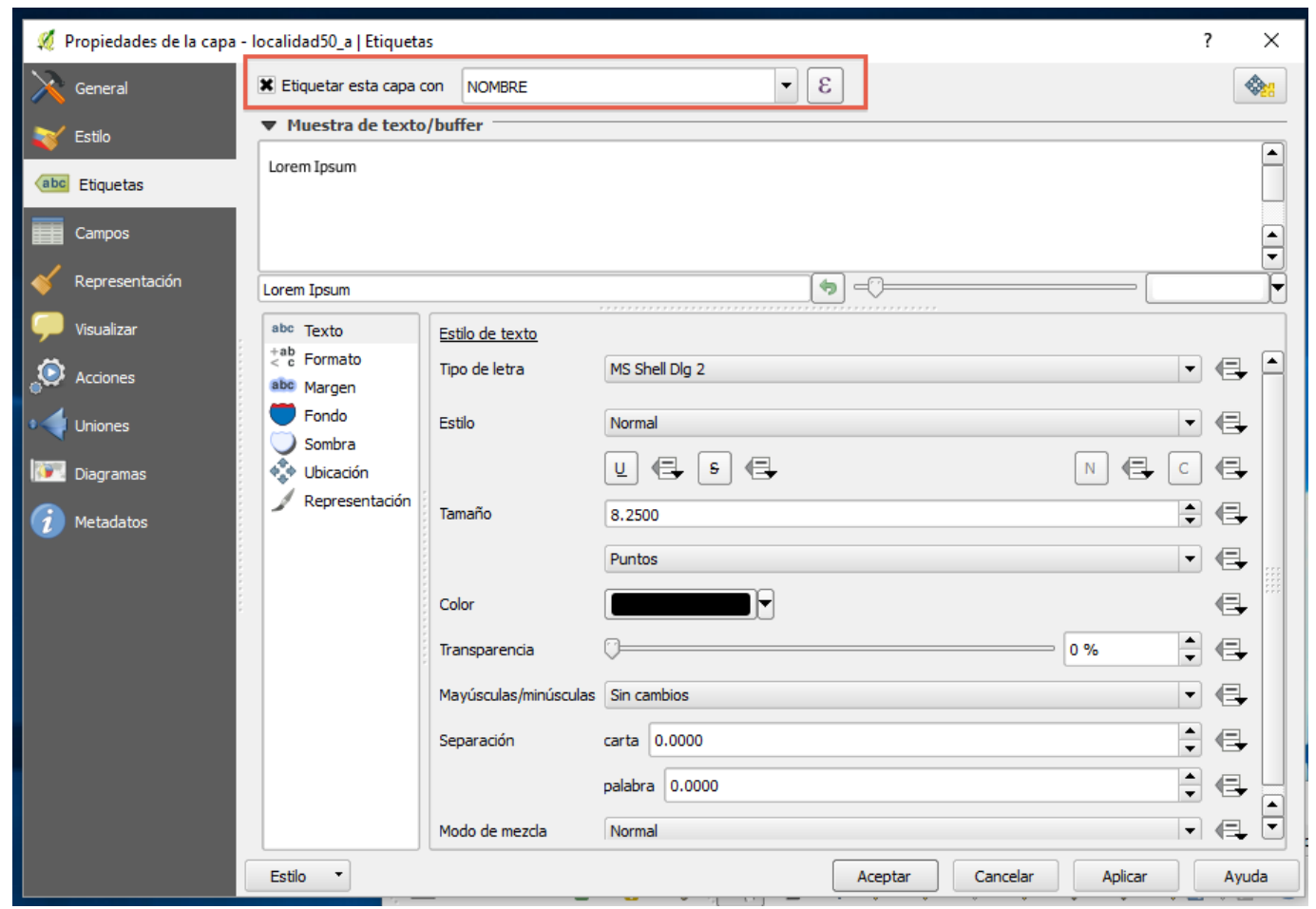

Figura 10: Cuadro de diálogo para mostrar las etiquetas

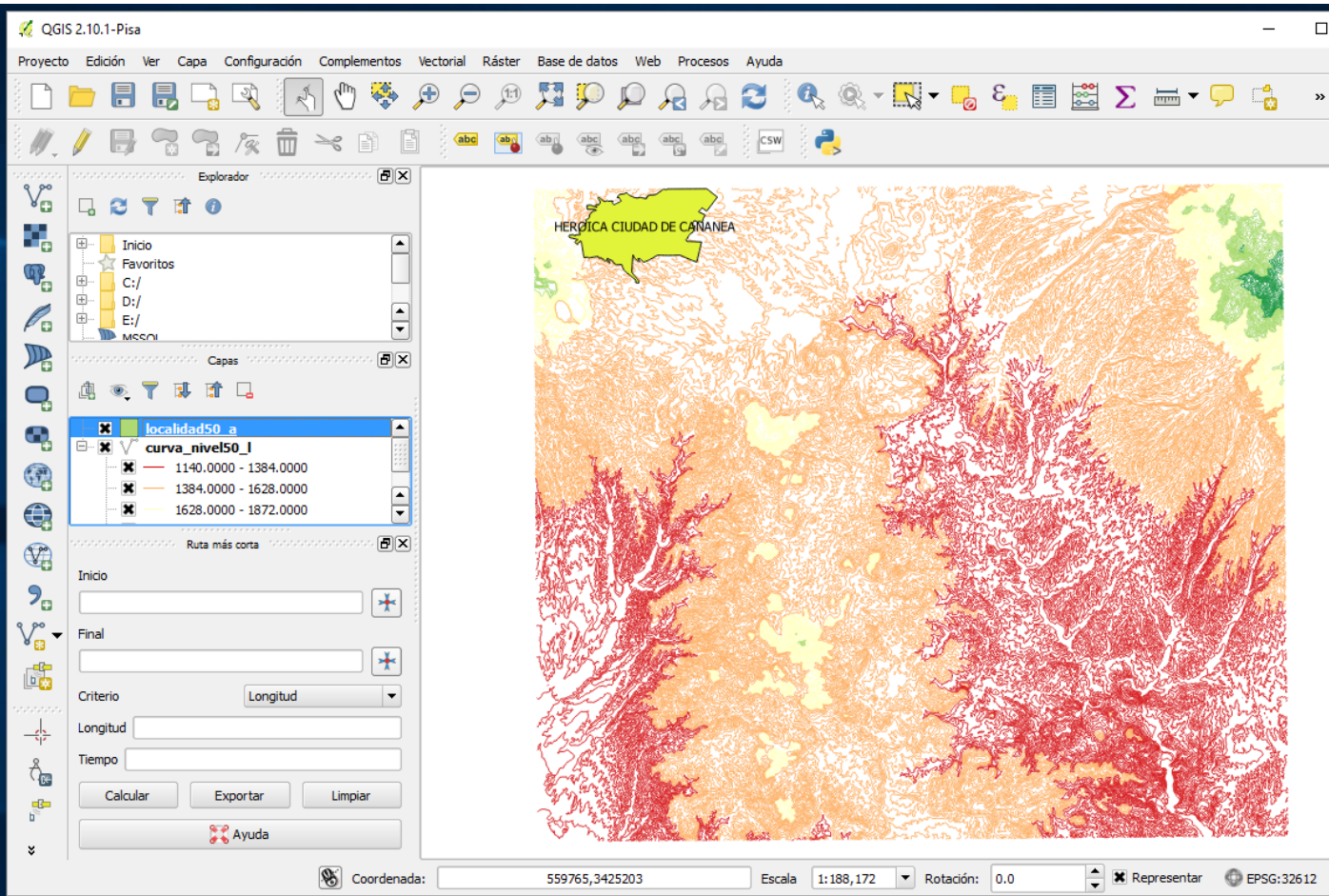

Figura 11: Mapa en el que se visualizan dos capas de información 
A continuación explicaremos la elección del Sistema de Referencia de Coordenadas. Hay que tomar en cuenta qué sistema de coordenadas se utilizó para producir el archivo. Usualmente junto con el archivo tipo .shp debe venir otro archivo con el mismo nombre, pero con otra extensión, .prj, en la cual se especifica el sistema de coordenadas utilizado. Por ejemplo, podemos buscar el archivo .prj correspondiente a las curvas de nivel. Este archivo es un archivo de texto, y se puede abrir con WordPad, TextEdit u otro procesador de textos sencillos. Este tipo de archivos no se debe modificar, solamente se debe consultar. En él se indica qué sistema de coordenadas hay que utilizar (en este caso, el sistema de coordenadas UTM zona 12N). En el caso del INEGI, además de los archivos prj viene un archivo de metadatos con extensión .txt en el cual vienen explicadas varias de las especificaciones técnicas. También viene adjunto el diccionario de datos topográficos, escala 1:50 000 (versión 2 ) en el cual se explican varios aspectos de las variables representadas.

\subsection{El Continuo de Elevaciones Mexicano}

Navegando en la página del INEGI [12] se pueden buscar también los datos de relieve continental. Uno de los productos que se pueden descargar es el Continuo de Elevaciones Mexicano, versión 3.0, (CEM 3.0). El usuario puede elegir descargar solamente una parte, por ejemplo la de Sonora. También se puede elegir una de las resoluciones disponibles, por ejemplo 120.

Al desempaquetar aparecen cinco archivos. El archivo que contiene los datos en formato de malla rectangular tiene extensión .bil, los otros archivos son auxiliares, tienen extensiones .xml, .hdr, .prj y .stx respectivamente. Abriendo el archivo .prj (el cual contiene la información de la proyección utilizada) se puede leer que se elaboró utilizando un sistema de coordenadas geográficas (longitud $=$ grados al este del meridiano de Greenwich y latitud = grados al norte del ecuador), con el esferoide (elipsoide) GRS 1980. Sobre este esferoide hablaremos más adelante. Véase la figura 12.

Sonora30_R120m.prj
GEOGCS["GCS_ITRF_1992",DATUM["D_ITRF_1992",SPHEROID
["GRS_1980",6378137.0,298.257222101]],PRIMEM["Greenwich",
0.0],UNIT["Degree",0.0174532925199433]]

Figura 12: Contenido de un archivo .prj en el cual se especifica que se utilizan coordenadas geográficas y el elipsoide GRS 1980

Para abrir el archivo con los datos en formato de malla rectangular utilizando QGIS se procede como sigue:

Primero se lanza la aplicación QGIS

Luego se va a la pestaña Capas, y en ella, Añadir capa ráster.

Se elige el archivo con extensión .bil

Utilícese el sistema de referencia de coordenadas EPSG 4019 correspondiente al elipsoide GRS 1980. 
El resultado debe desplegarse como en la figura 13.

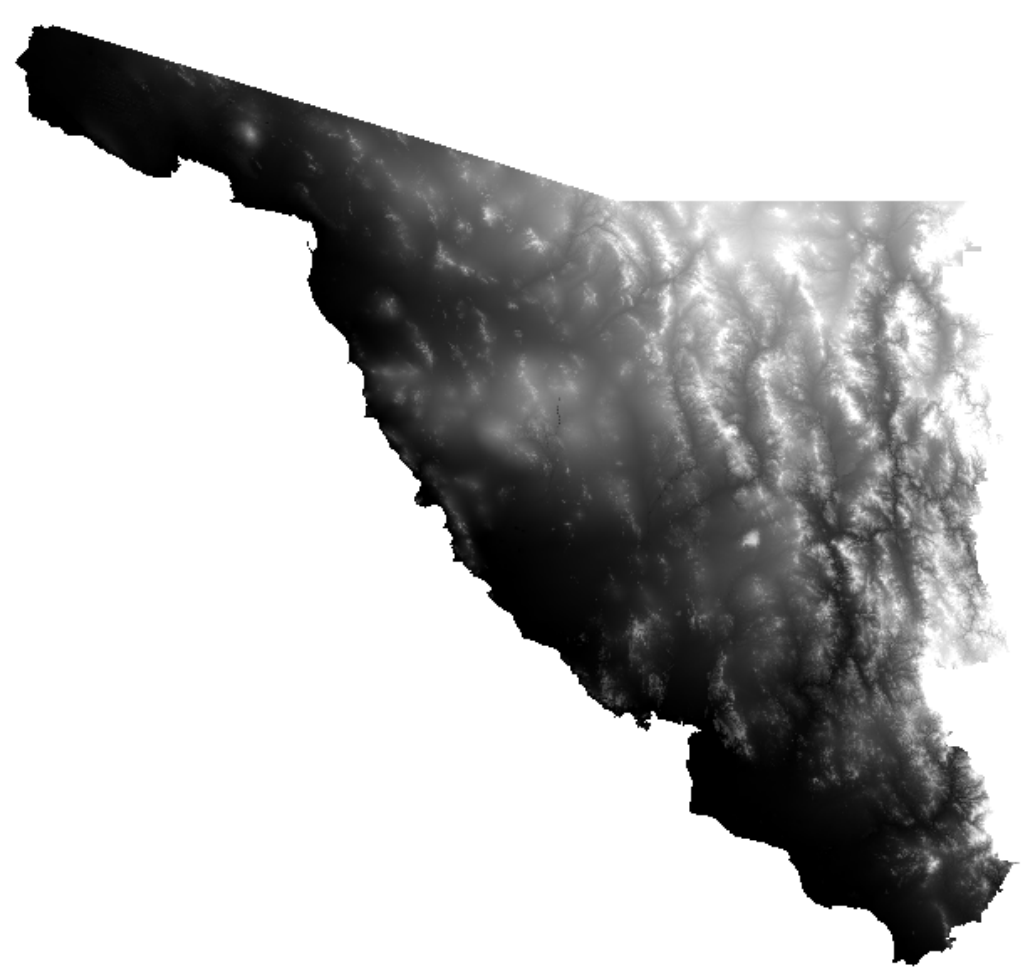

Figura 13: Continuo de Elevaciones Mexicano (Sonora)

Podemos modificar la forma de desplegar esta capa como sigue:

Elegir Capas, Propiedades, Estilo

Donde dice Tipo de renderizador cambiar Unibanda gris por Unibanda pseudocolor

Donde dice Generar nuevo mapa de color seleccionar la escala de colores Spectral. A continuación Clasificar, Aplicar, Aceptar.

El resultado debe verse como en la figura 14.

Si así lo deseamos, podemos consultar la elevación de cualquier punto en el mapa. Para ello hay que seleccionar la opción de Identificar objetos espaciales. El apuntador debe estar acompañado de una i. Al hacer click sobre un punto, QGIS nos dice la altura del mismo.

Cabe señalar que si solamente se tratara de visualizar y consultar la información, no sería necesario utilizar QGIS. El mismo INEGI, en su página beta [13] permite acceder a un sistema de información geográfica especial para trabajar con los datos del INEGI, se llama Espacio y Datos de México. Otra opción es el Mapa Digital de México, el cual se puede consultar en la página inicial [12]. Por otra parte, el INEGI no es la única fuente. La Comisión Nacional de Biodiversidad (Conabio) y la Comisión Nacional del Agua (Conagua) también están recabando y produciendo información geográfica en formatos digitales. 


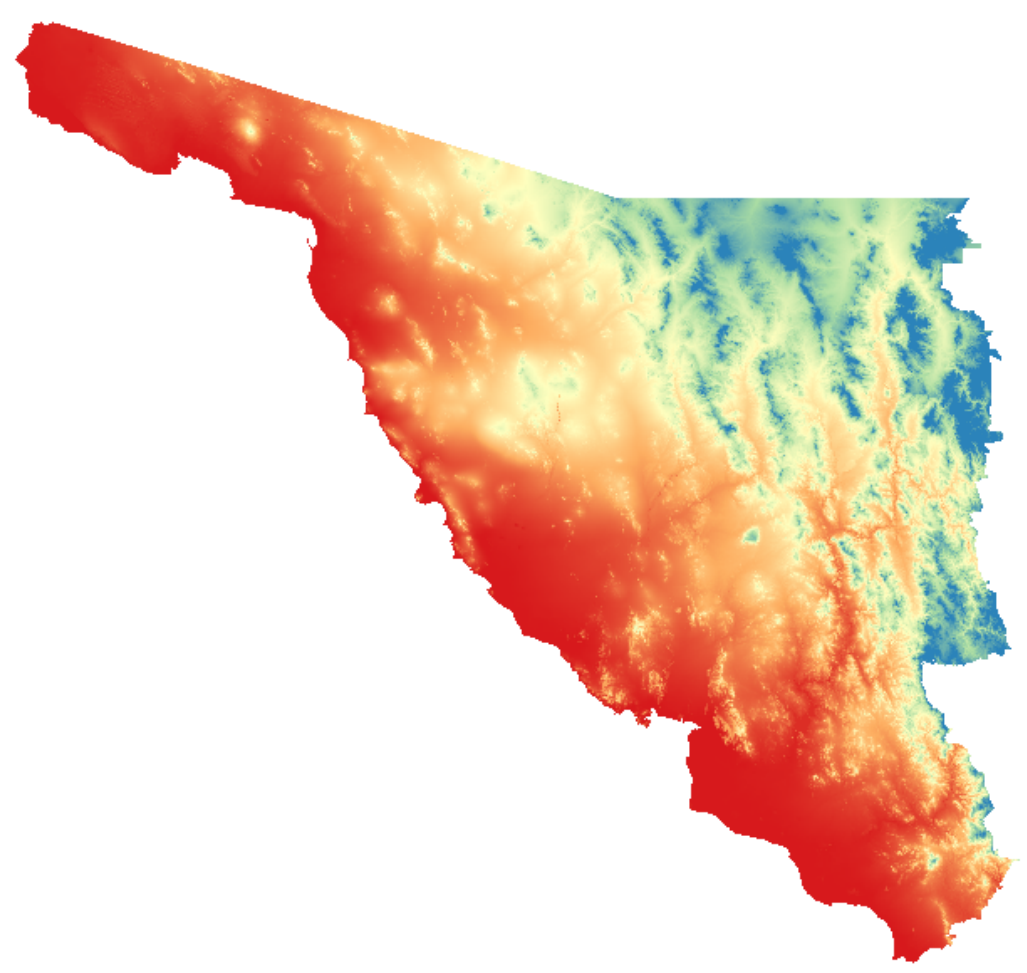

Figura 14: Continuo de Elevaciones, desplegado con escala de colores

Para saber más sobre los productos del INEGI recomendamos al lector visitar la página del mismo [12]. Cabe señalar que tienen asesores que prestan atención telefónica y en línea (chat).

\section{Datos vectoriales y datos en formato de malla rectangular}

A continuación vamos a comentar algunas características generales de archivos como los que discutimos en la sección anterior.

\subsection{Datos Vectoriales}

En un mapa bidimensional, tenemos básicamente tres maneras de representar un objeto espacial: mediante puntos, trayectorias poligonales o polígonos. Se dice que el tipo de representación es el tipo de 'geometría' del archivo de datos vectoriales. A continuación comentaremos algo sobre cada uno de ellos.

\subsubsection{Representación por puntos}

Si los objetos que queremos representar son muy pequeños en comparación con la extensión de la superficie terrestre en la cual queremos visualizarlos, puede ser conveniente representarlos por puntos. Cada punto tendrá asociadas dos coordenadas. Dependiendo del problema 
que nos interese tratar, elegimos variables cuyos valores registramos en cada uno de los objetos, además de las coordenadas. Así podemos formar dos bases de datos, una de ellas para las coordenadas de cada objeto, y otra para el resto de las variables de interés. Estas dos bases de datos estarán enlazadas, de manera que cada objeto esté registrado con un mismo identificador, lo cual permitirá integrar la información registrada en cada una de ellas.

Para fijar ideas, veamos un ejemplo. Supongamos que la vegetación se clasifica en dos tipos, viznaga y cactus. Y que haciendo un recorrido a lo largo y ancho de Sonora, encontráramos algunos ejemplares de uno y otro tipo. A cada ejemplar le asociaremos un número identificador, y registraremos las coordenadas de latitud y longitud, así como la especie a la cual pertenece. Mediante QGIS, esta información se puede capturar en un archivo tipo .shp como sigue:

Primero abrimos con QGIS un mapa de la región en la que estemos trabajando (en este caso utilicemos el mapa de elevaciones de Sonora).

Crearemos un archivo vectorial en el cual capturar los datos. Esto podemos hacerlo seleccionando sucesivamente las opciones de Capa, Nueva, Nueva capa de archivo shape.

Aparecerá un cuadro de diálogo, en el cual tenemos que precisar qué tipo de representación geométrica se utilizará. Las opciones son Punto, Línea, Polígono. Seleccionemos Punto.

También hay que especificar el sistema de referencia de coordenadas. Seleccionemos EPSG 4019 (GRS 1980). A priori, solamente aparece un campo en la lista de atributos, tiene el nombre id, toma valores enteros y se usa como identificador. Podemos añadir otros campos a la lista de atributos, datos de texto, números enteros, decimales o fecha. Introduzcamos un campo llamado Especie cuyos valores sean de texto. Para ello escribamos Especie en el cuadro de nombre de Nuevo atributo, seleccionemos la opción Datos de texto, y oprimamos el botón Añadir a la lista de atributos. Luego habrá que ponerle nombre al archivo de datos vectoriales, digamos Especies. Una vez creado el archivo, vamos a capturar los datos. Es necesario indicar que queremos modificar la capa, para ello, en la pestaña de Capa elegimos Conmutar edición.

Ahora vamos a la pestaña Edición, y en ella seleccionemos Añadir objeto espacial. Ahora llevemos el cursor a las coordenadas donde están las observaciones de los ejemplares, hagamos click justo encima de donde está la observación. Aparecerá un cuadro de diálogo en el cual se nos pide llenar los campos del ejemplar que estamos registrando. En este caso son id (un número identificador) y Especie (el campo que añadimos nosotros). Supongamos que la primera observación es una viznaga, entonces en id capturamos el número 1 y en Especie capturamos el nombre de la especie. Supongamos que el ejemplar número dos es un cactus, hacemos lo mismo en el punto correspondiente. Hacemos lo mismo con todas las observaciones que tengamos registradas, al final desactivamos la posibilidad de edición volviendo a hacer click en donde dice Conmutar edición y guardamos los cambios. La nueva capa debe quedar visible.

Podemos representar de manera diferente a las observaciones según su especie. En la pestaña de Capa seleccionamos Propiedades, Estilo, y cambiemos la opción de renderizar 
mediante Símbolo único, por la opción de renderizar Basado en reglas.

En el cuadro de diálogo hagamos click en el símbolo de + (añadir regla). En donde dice Filtrar hagamos click en el botón con puntos suspensivos, seleccionemos Campos y valores. Ahi seleccionemos Especie e indiquemos que cargue los valores únicos que tenemos. Luego, en el cuadro de Expresión escribamos el nombre del campo (en este caso Especie) luego $=$, y luego vamos a viznaga y hagamos doble click en ella. A continuación seleccionemos el símbolo y el color. Luego oprimamos el botón Aceptar. Debe quedar añadida una regla. Repitamos el proceso para añadir otra regla para los cactus. Una vez añadidas las reglas, oprimimos los botones Aplicar, Aceptar. El resultado debería ser como el de la figura 15.

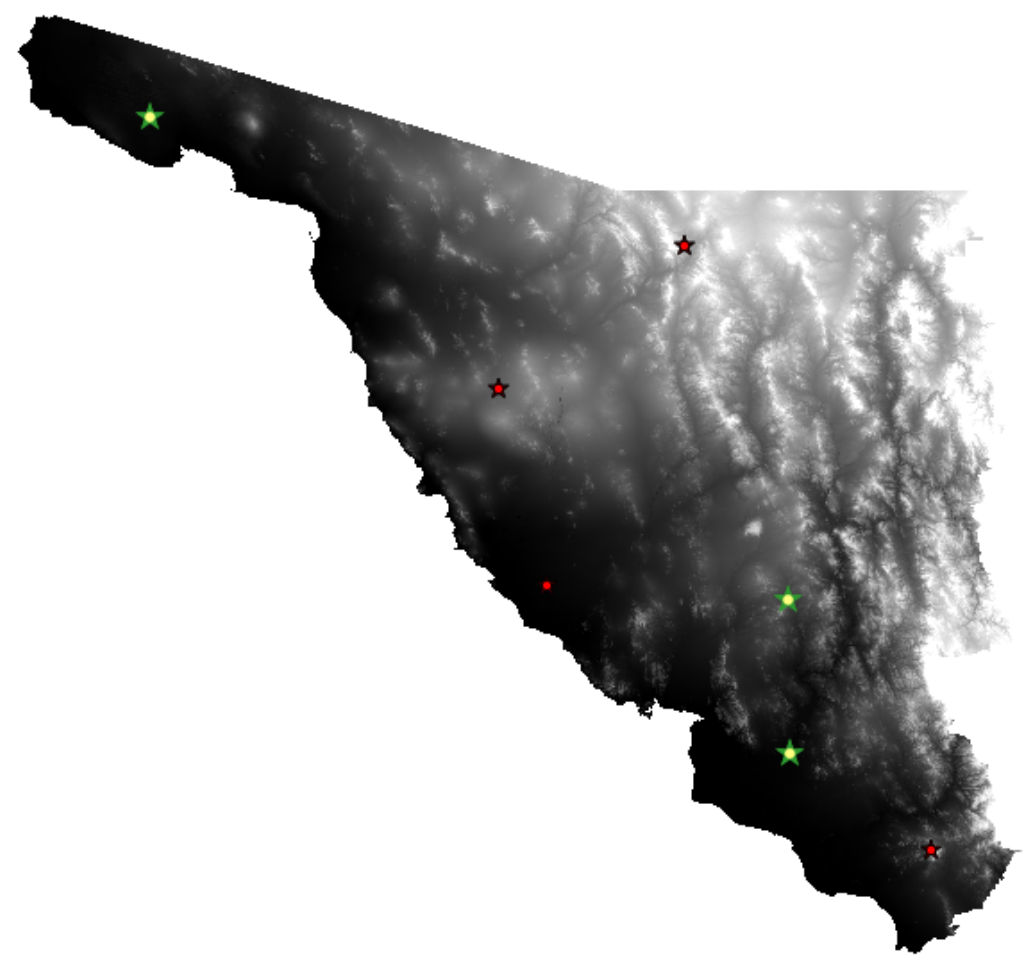

Figura 15: Capa de Especies, sobrepuesta a la capa de Elevaciones

Otros objetos, como las localidades pequeñas o las minas también pueden representarse por puntos. Ahora bien, cada capa tiene asociada una base de datos, dicha base está en el archivo con extensión .dbf que se crea junto con el archivo .shp y con el archivo .pjr. Podemos consultarla en QGIS cargando la capa y luego seleccionar Abrir tabla de atributos en la pestaña Capa.

\subsubsection{Representación por trayectorias poligonales}

Otros objetos espaciales se representan más adecuadamente por líneas poligonales. Por ejemplo, las carreteras, los ríos y las curvas de nivel. El proceso de captura es parecido al 
de los puntos, solamente que para definir un objeto debemos capturar varios puntos (los vértices o nodos de la trayectoria poligonal). Ahora bien, una trayectoria poligonal tiene un rasgo que no tienen los puntos, y es la longitud del recorrido.

\subsubsection{Representación por polígonos}

Otros objetos espaciales se representan más adecuadamente por polígonos cerrados, por ejemplo, cuencas hidrográficas, cuerpos de agua, áreas de cultivo y grandes localidades. Estos objetos también tienen asociada el área del polígono con el que lo representamos. Veamos un ejemplo.

En la página del INEGI donde buscamos la carta topográfica de Cananea edición 2016, veamos la carta topográfica edición 2000. Viene en cinco formatos a escoger. Descarguemos el archivo con extensión tif. Como antes, en realidad descargamos un archivo empaquetado. Al desempaquetar obtenemos tres archivos. Uno de ellos es un archivo de texto, otro es el archivo tif, y el otro es tfw.

Para abrirlo, en QGIS procedemos como sigue:

Capa, Añadir capa, Añadir capa ráster

Seleccionamos el archivo h12b53 con extensión tif

Seleccionamos el sistema de coordenadas WGS 84 / UTM 12N

QGIS debe desplegar el mapa. Es como un pdf pero en él podemos ver las coordenadas de cada punto.

Hagamos un acercamiento en la esquina superior izquierda de la imagen. Vayamos al cuerpo de agua llamado Concentradora Vieja

A continuación vamos a crear un archivo de datos vectoriales en el cual quede registrado un polígono, uno que represente a este cuerpo de agua.

Primero creamos el archivo en el cual vamos a capturar el polígono

Capa, Crear capa, Nueva capa de archivo shape

Aparecerá un cuadro de diálogo.

En la opción de Tipo elegimos Polígono

Por simplicidad y brevedad ahora no agregaremos ningún nuevo atributo. Elegimos Aceptar 


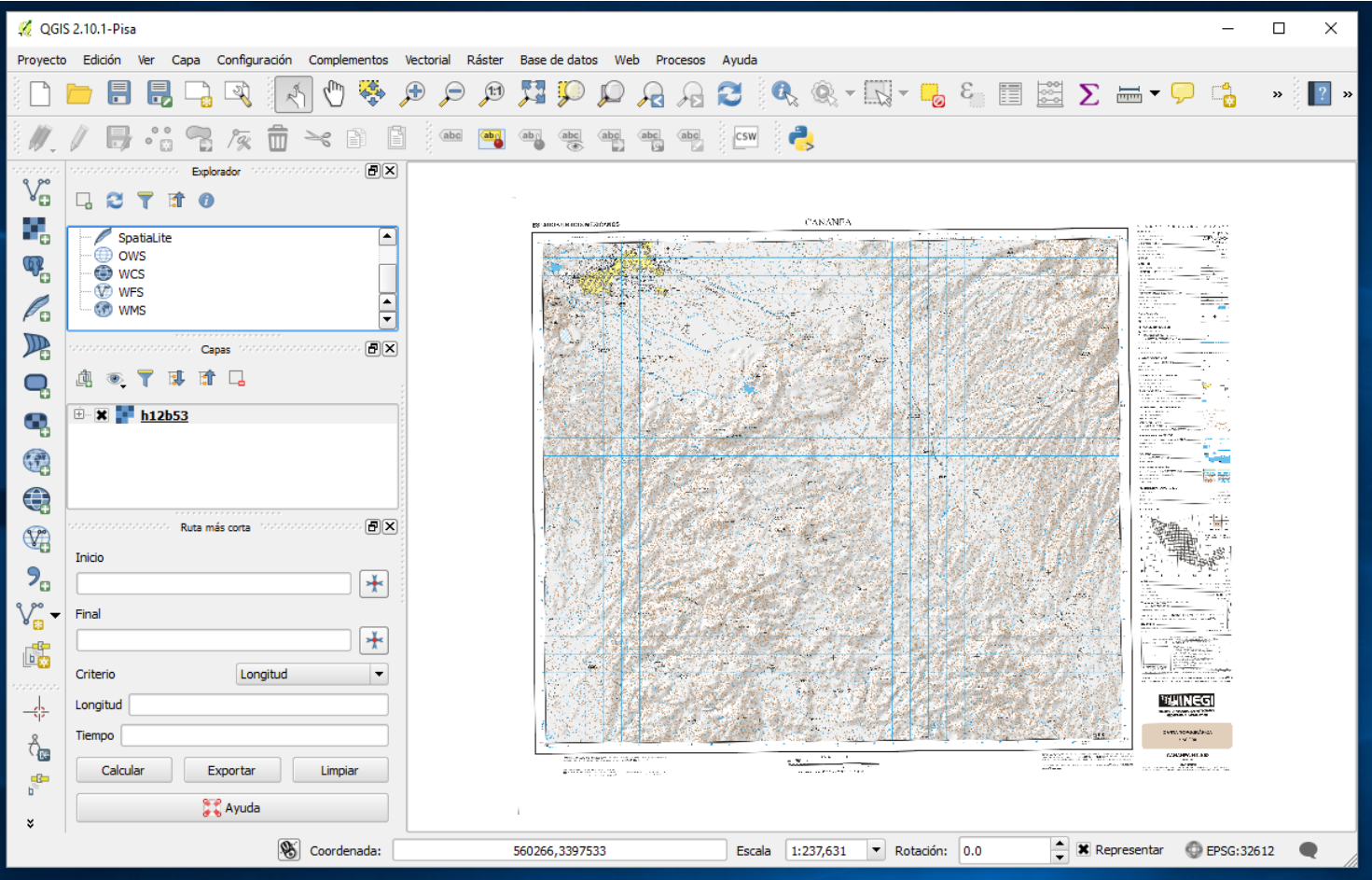

Figura 16: Una carta topográfica en formato tif

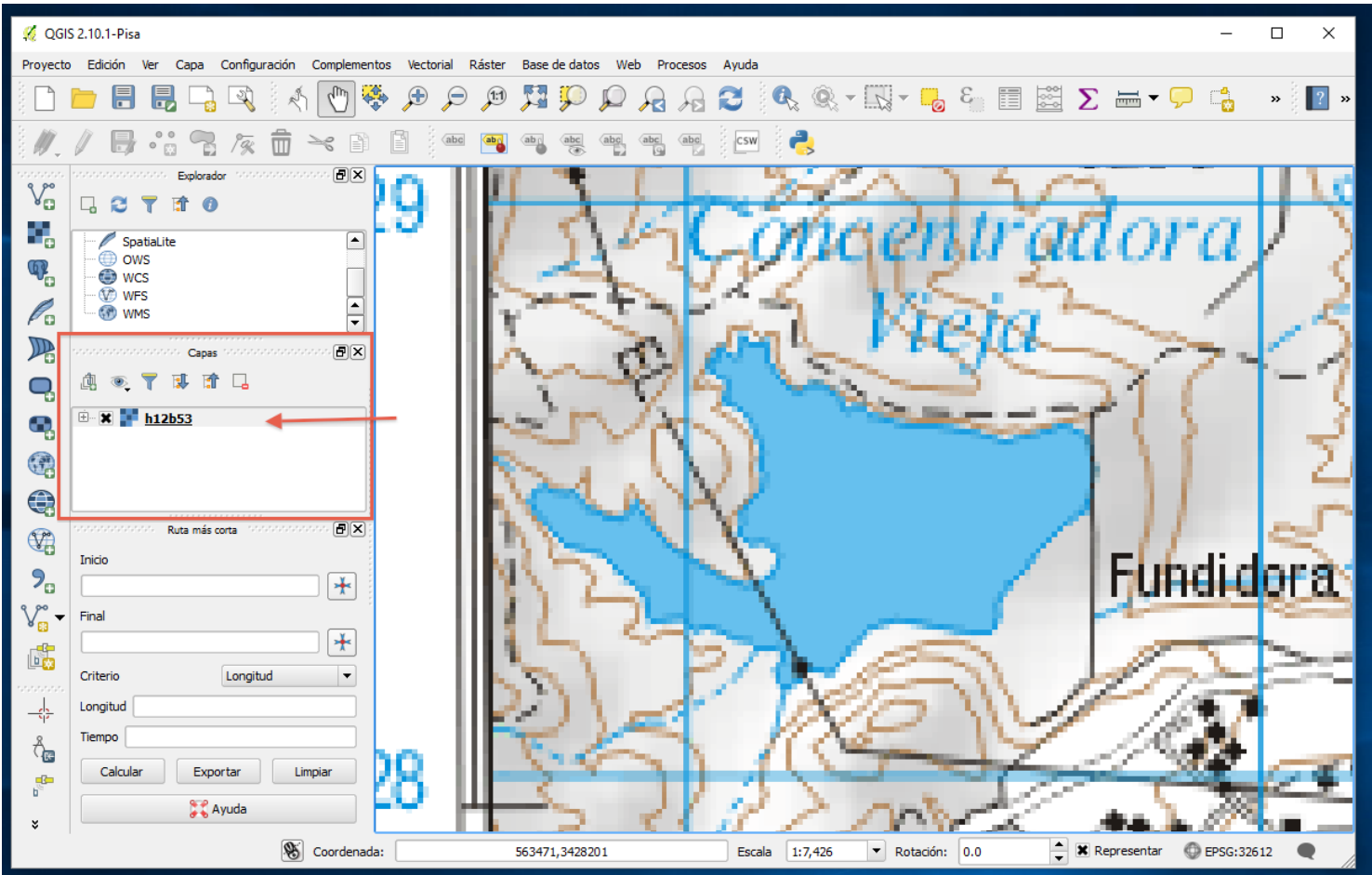

Figura 17: A partir de un mapa crearemos un archivo tipo shp 


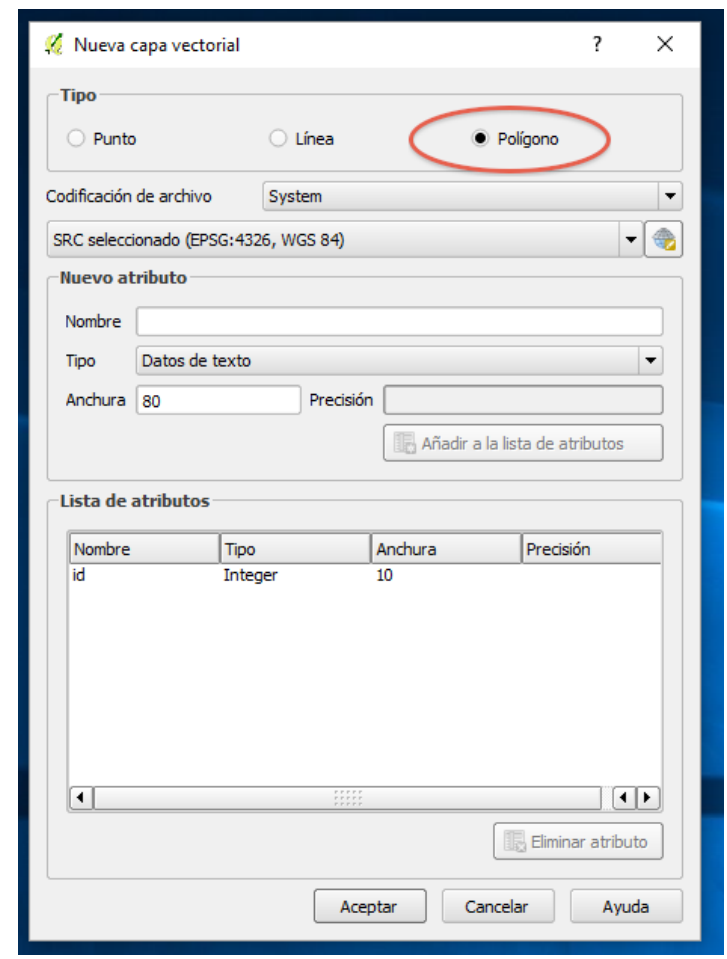

Figura 18: Creación de un archivo de datos vectoriales

Elegimos el nombre del archivo, digamos Laguna, a continuación salvamos el archivo recién creado.

En la ventana de Capas ubicamos el ratón en el archivo recién creado y hacemos click con el botón izquierdo para seleccionarlo, luego click con el botón derecho, aparece un menú local, en él seleccionamos Conmutar edición.

Elegimos la opción de Aadir objeto espacial

Hacemos click con el botón izquierdo del ratón en un punto. Vamos a otro y volvemos a hacer click. Así sucesivamente hasta recorrer el polígono que estamos capturando. Para finalizar hacemos click con el botón derecho del ratón. Aparecerá un cuadro de diálogo para anotar el número identificador del polígono que estamos capturando. Llenamos el campo id con el número 1 (es el primer polgígono que capturamos), luego Aceptar. A continuación podrámos capturar un segundo polígono, pero en este caso no o vamos a a hacer. Terminada esta etapa, guardamos los cambios en la capa. Como ya no vamos a hacer más cambios, salimos del modo de edición haciendo click en el botón de Conmutar edición 


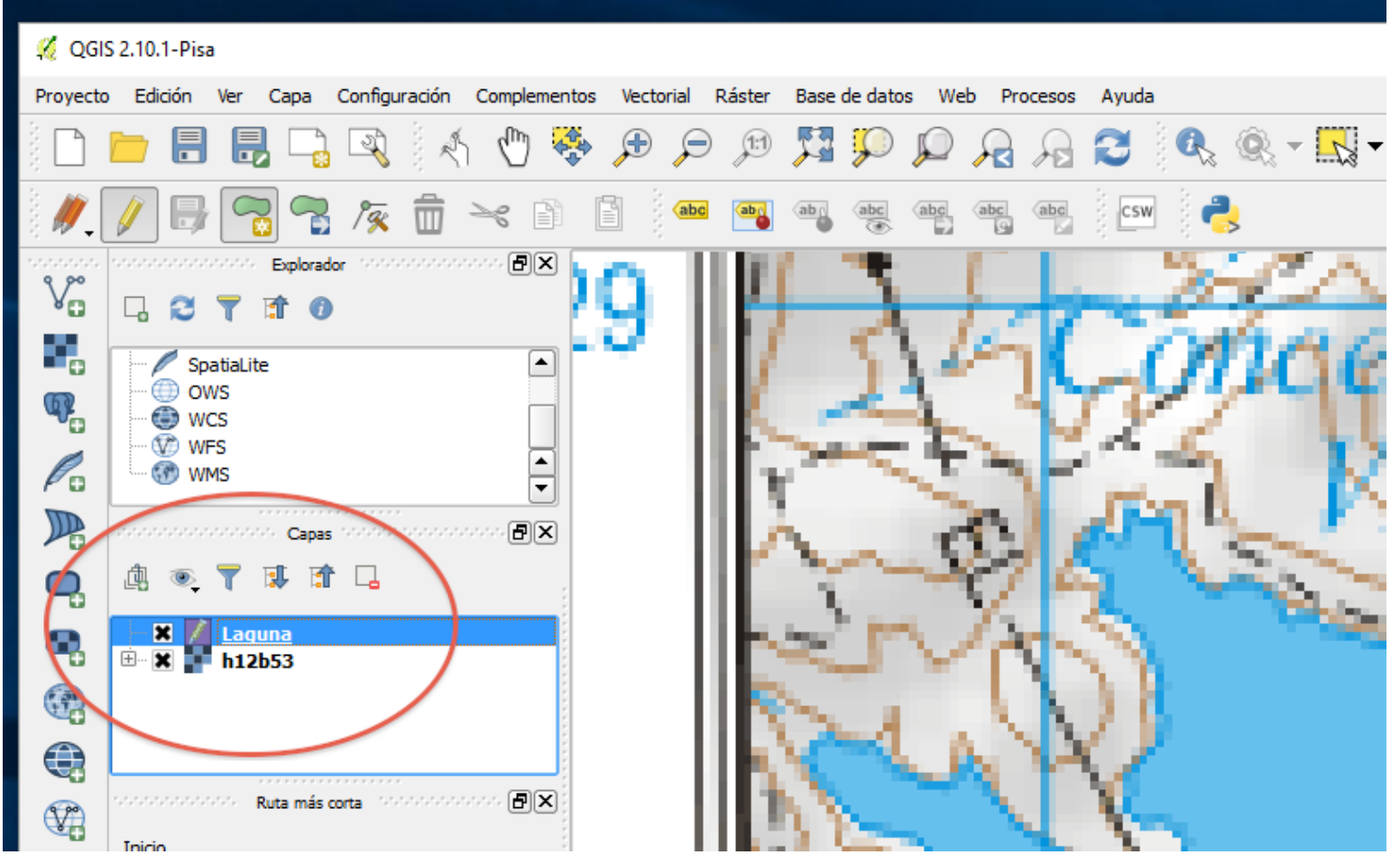

Figura 19: La creación del archivo Laguna se refleja en la ventana Capas

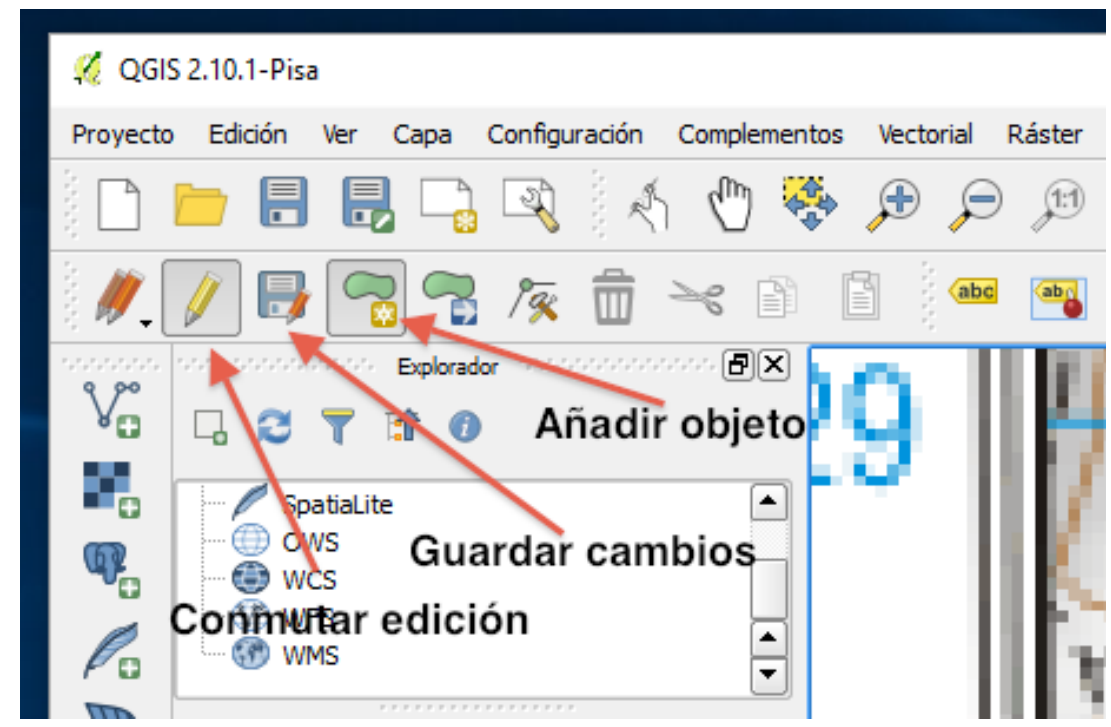

Figura 20: Botones usados en la edición

\subsection{Datos en formato de malla rectangular}

Otra manera de representar información geográfica es mediante archivos tipo mallas rectangulares (son más conocidos como grid o ráster). Podemos pensar que el mapa abarca una región rectangular, la cual está dividida en celdas pequeñas (como los pixeles de una fotografía). Cada celda tiene asociado un número, el cual puede ser la elevación sobre el nivel 
del mar, puede ser temperatura, precipitación u otra variable de interés. Entre los archivos más simples que se usan para representar información de este tipo, están los archivos con extensión .xyz, estos archivos no son más que archivos de texto que guardan la información en tres columnas, se suelen utilizar las dos primeras para las coordenadas del centro de la celda, y la tercera columna para el valor de la variable z representada en el mapa. Construyamos un ejemplo sencillo. Con un editor de texto (como WordPad o TextEdit) elaboremos un archivo que podría tener extensión .txt, pero guardémoslo con la extensión .xyz, para que QGIS pueda reconocerlo como un archivo de datos en formato de malla rectangular. Capturemos los números que se muestran en la tabla 1.

Tabla 1: Ejemplo de datos que contiene un archivo .xyz

$\begin{array}{lll}565000 & 3427000 & 10 \\ 566000 & 3427000 & 11 \\ 567000 & 3427000 & 12 \\ 568000 & 3427000 & 14 \\ 569000 & 3427000 & 13 \\ 570000 & 3427000 & 11 \\ 565000 & 3428000 & 10 \\ 566000 & 3428000 & 11 \\ 567000 & 3428000 & 13 \\ 568000 & 3428000 & 17 \\ 569000 & 3428000 & 16 \\ 570000 & 3428000 & 14 \\ 565000 & 3429000 & 10 \\ 566000 & 3429000 & 12 \\ 567000 & 3429000 & 16 \\ 568000 & 3429000 & 18 \\ 569000 & 3429000 & 17 \\ 570000 & 3429000 & 15\end{array}$

Cabe señalar que en este ejemplo las celdas son de un kilómetro cuadrado, pues las coordenadas UTM están en metros, y la distancia entre los centros de dos celdas adyacentes es de 1000 metros. Si lanzamos QGIS y abrimos el archivo de datos vectoriales con las localidades representadas como área, luego cargamos este archivo con una secuencia Capa, Añadir capa ráster, y finalmente ajustamos la transparencia de la última capa, el resultado será como el que muestra en la figura 8.

Para saber más sobre cómo utilizar QGIS remitimos al lector a los documentos y manuales que están en la página de QGIS [16]. Puede que esté demás, pero recomendamos al lector consultar también la documentación en inglés. 


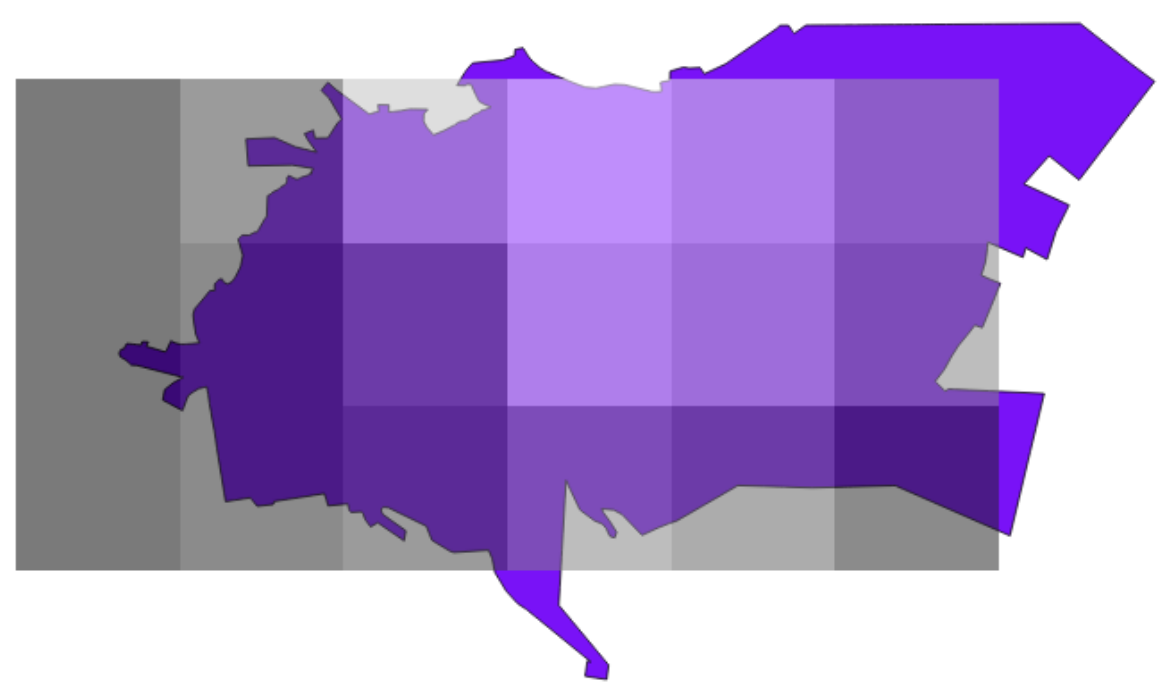

Figura 21: Capa de un archivo en formato xyz, sobrepuesta a la capa vectorial de localidades 50 a

\section{Sobre las coordenadas utilizadas}

\subsection{El elipsoide GRS 1980}

Un primer modelo de la superficie terrestre es una esfera. Sin embargo, para fines de la elaboración de mapas digitales, se utilizan otros modelos, particularmente el de un 'esferoide' el cual es una superficie de revolución que se obtiene al rotar una elipse alrededor del semieje menor. Uno de ellos se conoce como Geodetic Reference System (GRS) y fue calculado en 1980. De acuerdo con Snyder [5], el radio ecuatorial tiene una longitud de

$$
a=6,378,137 \text { metros }
$$

El radio polar tiene una longitud de

$$
b=6,356,752.3 \quad \text { metros }
$$

El factor de achatamiento $f$ es un número que intuitivamente nos indica en qué porcentaje se reduce el radio ecuatorial para tener la longitud del radio polar. Es decir, se relaciona con $a$ y con $b$ mediante la fórmula

$$
a(1-f)=b
$$


Es de aproximadamente 1 parte de cada 300, o mejor aún

$$
f=\frac{1}{298.257}
$$

Cabe señalar que estas medidas resultan de ajustar el modelo del elipsoide globalmente a la superficie terrestre, sin embargo, es posible que para modelar localmente, otras medidas den un ajuste mejor.

\subsection{Sobre el sistema de coordenadas UTM}

Para introducir el sistema de coordenadas que utiliza la proyección Universal Transversal de Mercator (UTM) conviene recordar algunos hechos que nos ayudarán a comprender lo que nos vamos a encontrar cuando trabajemos con este sistema de coordenadas.

Tomemos en cuenta que al tratar de representar puntos de una superficie con curvatura positiva (como la esfera) en una superficie plana, necesariamente habrá propiedades que se pierdan, especificamente:

Puede que no se preserven las distancias

Puede que no se preserven los ángulos.

Puede que no se preserven las áreas.

Una manera de generar un mapamundi es proyectar los puntos de una esfera sobre un cilindro tangente a la esfera en los puntos del ecuador, y luego desenrollar el cilindro. El ecuador va a dar a una línea horizontal. Los círculos paralelos al ecuador irán a dar a líneas horizontales. Los círculos máximos que pasan por los polos (los meridianos) van a dar a líneas verticales. Esta proyección cilíndrica es la proyección de Mercator. Cerca del ecuador, las deformaciones son pequeñas, lejos del ecuador (como en Groenlandia) las deformaciones son mayores.

Una variante de esta proyección se obtiene cambiando la posición del cilindro. Se puede poner tangente a un meridiano. Cerca de ese meridiano, las deformaciones serán pequeñas. Podemos dividir la superficie terrestre en regiones separadas por los meridianos que van desde $180^{\circ} \mathrm{W}$ hasta $180^{\circ} \mathrm{E}$ cada $6^{\circ}$ de longitud. La tierra quedará dividida en 60 regiones. Dado cualquier punto de tierra firme, podemos ubicarlo en una de estas regiones. Las zonas UTM están delimitadas al este y al oeste por estos meridianos. Hacia el norte y hacia el sur la proyección transversal de Mercator no se usa con latitudes mayores a $84^{\circ}$, cerca de los polos se utilizan otras proyecciones. Para asignar las coordenadas UTM también se diferencia si el punto está en el hemisferio norte o sur. En el caso de puntos en el hemisferio norte, se le asignan las coordenadas 


$$
(x, y)=(500000,0)
$$

al punto que esté en el ecuador en uno de los meridianos centrales de cada una de las 60 zonas UTM. En el caso de puntos en el hemisferio sur, a dicho punto se le asignan coordenadas

$$
(x, y)=(500000,10000000)
$$

Las unidades se dan en metros, y podríamos decir que utilizamos 120 cartas para cubrir a la esfera que modela a la superficie terrestre, salvo dos discos centrados en los polos. En todos los casos, el eje $X$ apunta hacia el este, el eje $Y$ apunta hacia el norte, y al interior de cada zona UTM todas las coordenadas son positivas.

Ahora bien, si el cilindro es tangente a la esfera en un meridiano central, cerca de los puntos que están en el meridiano central las deformaciones son pequeñas, mientras que las deformaciones crecen a medida que nos alejamos de dicho meridiano central. Sin embargo, en promedio, las deformaciones tienden a alargar las distancias en cada zona. Para compensar este hecho, se aplica un factor de reducción de 0.9996 lo cual tiene el efecto de achicar las distancias en el meridiano central, agrandarlas menos en los meridianos extremos, y en promedio las distorsiones se compensan entre unos y otros puntos de la misma zona.

Además se realizan otros ajustes para utilizar el modelo del elipsoide en lugar del modelo de la esfera, lo cual complica las fórmulas que describen la relación entre las coordenadas UTM y las coordenadas de latitud y longitud.

Para entender un poco más las deformaciones a las cuales hacemos referencia enfocaremos el asunto desde dos perspectivas: una será más geométrica y cualitativa, aunque se tomará el modelo de la esfera en lugar del modelo del elipsoide. La otra será más cuantitativa y numérica, dejando que las computadoras hagan las cuentas y simplemente observemos los números que nos dan.

Consideremos una región en una esfera delimitada por dos meridianos y dos paralelos, los cuales estén separados por un grado de longitud y de latitud, respectivamente.

Nótese que esta región:

Está delimitada por una curva suave por pedazos, que tiene ángulos rectos en sus cuatro vértices.

Los segmentos de curva que unen dos vértices consecutivos tienen longitudes diferentes. Dos de ellos son arcos de círculos paralelos que tienen radios diferentes. Para ser más precisos, el lado norte mide menos que el lado sur (en el hemisferio en el cual estamos trabajando). 


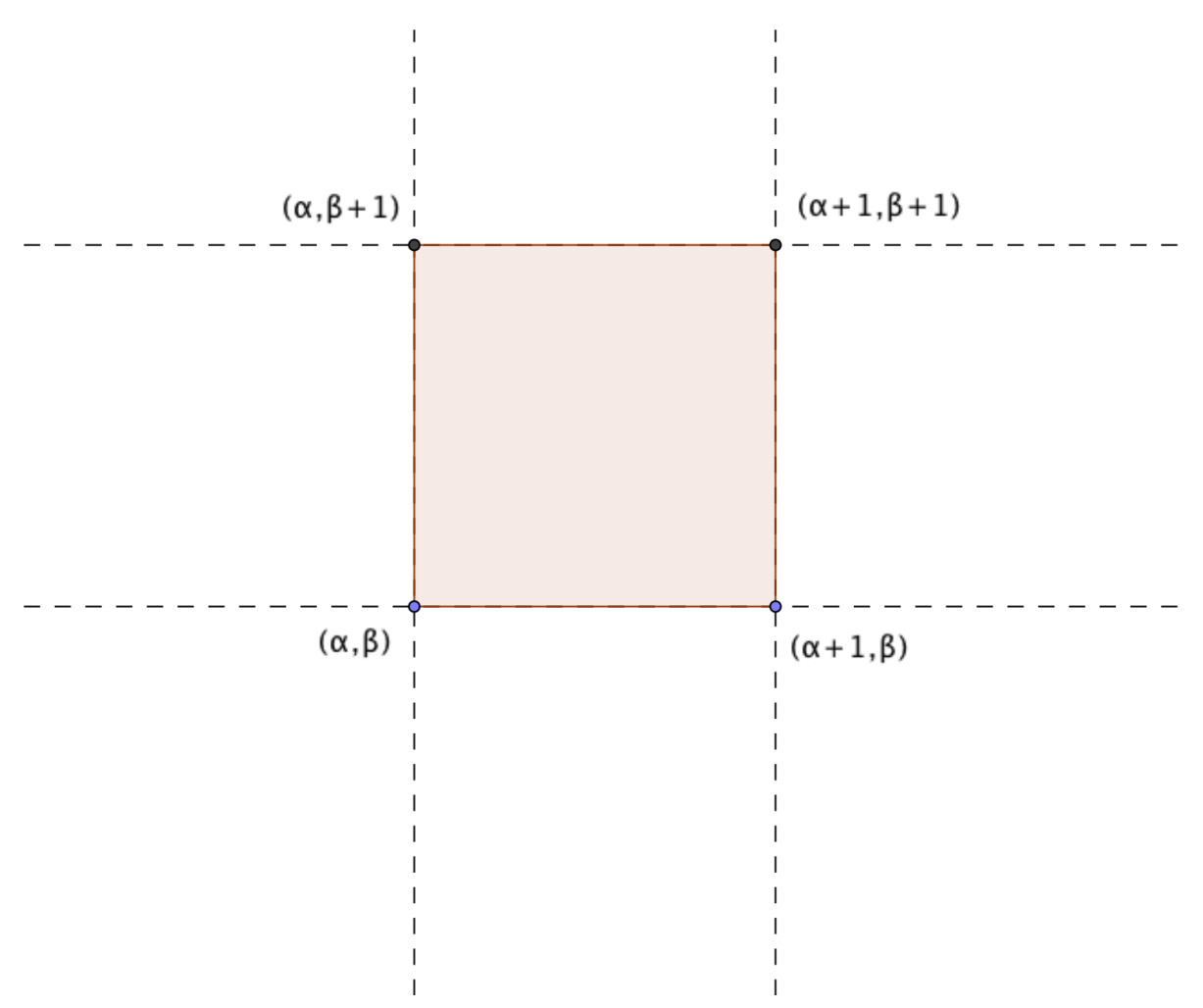

Figura 22: Región delimitada por meridianos de $\alpha$ y $\alpha+1$ grados de longitud, y por paralelos de $\beta$ y $\beta+1$ grados de latitud

Consideremos la proyección de la esfera sobre un cilindro que sea tangente a lo largo de un meridiano. De todos los meridianos, el único que va a dar a una línea recta es el meridiano de tangencia. Los demás meridianos van a dar a otro tipo de curvas. Esto tiene como consecuencia que partiendo de latitudes diferentes, incrementos de un grado de longitud en la esfera, producen incrementos de longitud desiguales en el cilindro (y por lo tanto en el mapa).

Ahora consideremos una sucesión finita de puntos que estén en un mismo paralelo (a una misma latitud).

Supongamos que el punto medio de la hilera está en el meridiano donde el cilindro es tangente a la esfera. En la esfera los puntos están en una circunferencia. Cerca del meridiano de tangencia, proyectar sobre el cilindro es como proyectar sobre el plano tangente común a la esfera y al cilindro. Sabemos que al proyectar una circunferencia 'inclinada' sobre un cilindro nos produce una elipse. La imagen de nuestra hilera de puntos se parecerá a un segmento de elipse. Sin embargo, si la hilera estuviera en el ecuador, su imagen sería un segmento de recta. Dado que el ecuador va a jugar el papel del eje $X$ para los puntos que estamos considerando en el hemisferio norte, esto quiere decir que las ordenadas de los puntos de la imagen de la hilera que está en un paralelo, tienen un mínimo local al cruzar el meridiano central. 
$(\alpha, \beta) \quad(\alpha+1, \beta) \quad(\alpha+2, \beta) \quad(\alpha+3, \beta) \quad(\alpha+4, \beta)$

Figura 23: Una sucesión finita de puntos sobre un mismo paralelo

Ahora veamos los números correspondientes a puntos seleccionados. Vamos a utilizar una aplicación en línea [10] que convierte las coordenadas de latitud y longitud en coordenadas UTM. Comencemos con una hilera de puntos que pertenecen al paralelo de $30^{\circ}$ de latitud norte, que van desde los $114^{\circ}$ hasta los $108^{\circ}$ de longitud oeste. Veamos en qué zona UTM están.

1. La zona 1 va desde los $180^{\circ} \mathrm{W}$ hasta los $174^{\circ} \mathrm{W}$.

2. La zona 2 va desde los $174^{\circ} \mathrm{W}$ hasta los $168^{\circ} \mathrm{W}$.

3. La zona 3 va desde los $168^{\circ} \mathrm{W}$ hasta los $162^{\circ} \mathrm{W}$.

En general, para $k=1, \ldots, 30$ la $k$-ésima zona UTM va desde los $180-6(k-1)$ grados oeste hasta los $180-6 k$ grados oeste.

En particular, la zona 12 UTM va desde los $114^{\circ} \mathrm{W}$ hasta los $108^{\circ} \mathrm{W}$.

En la zona 12 UTM Norte el meridiano central es el de $111^{\circ} \mathrm{W}$. Comprobamos que en él, las ordenadas $Y$ de las coordenadas UTM alcanzan un valor mínimo (si los puntos pertenecen a un mismo paralelo). 
Tabla 2: Coordenadas UTM de puntos en el paralelo $30^{\circ} \mathrm{N}$

\begin{tabular}{llll}
\hline Longitud W & Latitud N & UTM zona $12(\mathrm{X})$ & UTM zona $12(\mathrm{Y})$ \\
\hline 114 & 30 & 210590.3 & 3322575.9 \\
113 & 30 & 307084.8 & 3320469.2 \\
112 & 30 & 403549.8 & 3319206.8 \\
111 & 30 & 500000.0 & 3318785.3 \\
110 & 30 & 596450.1 & 3319206.2 \\
109 & 30 & 692915.1 & 3320469.2 \\
108 & 30 & 789409.6 & 3322575.9 \\
\hline
\end{tabular}

Comparemos lo que sucede en un paralelo más cercano al ecuador. Esperamos que la curva de las ordenadas sea más parecida a un segmento de recta que en un paralelo más lejano del ecuador.

Tabla 3: Coordenadas UTM de puntos en el paralelo $15^{\circ} \mathrm{N}$

\begin{tabular}{llll}
\hline Longitud W & Latitud N & UTM zona $14(\mathrm{X})$ & UTM zona 14 $(\mathrm{Y})$ \\
\hline 102 & 15 & 177349.0 & 1660513.6 \\
101 & 15 & 284946.9 & 1659297.7 \\
100 & 15 & 403549.8 & 1658568.8 \\
99 & 15 & 500000.0 & 1658325.9 \\
98 & 15 & 607512.2 & 1658568.8 \\
97 & 15 & 715053.0 & 1659297.7 \\
96 & 15 & 822650.9 & 1660513.6 \\
\hline
\end{tabular}

Comparemos los datos de ambas tablas desde la persepectiva de cada uno de los sistemas de coordenadas.

En ambos casos tenemos una hilera de puntos que recorre el ancho de la banda delimitada por la zona UTM.

Desde el punto de vista de las coordenadas de longitud-latitud, en ambos casos el ancho de la banda es de $6^{\circ}$ de longitud.

Desde el punto de vista de las coordenadas UTM (las cuales están en metros), es mayor el intervalo que recorren los puntos del paralelo $15^{\circ} \mathrm{N}$ que el intervalo que recorren los puntos 
del paralelo $30^{\circ} \mathrm{N}$. Esto así tenía que ser, pues desde la esfera, el paralelo $15^{\circ} \mathrm{N}$ es parte de una circunferencia que tiene un radio mayor que la circunferencia correspondiente al paralelo $30^{\circ}$ N. En este aspecto, las coordenadas UTM reflejan mejor lo que sucede en la esfera que las coordenadas de latitud y longitud.

Ahora vamos a México. Está contenido en una región delimitada al norte por el paralelo $32^{\circ} 43^{\prime} \mathrm{N}$ y al sur por el paralelo $14^{\circ} 32^{\prime} \mathrm{N}$, al este por el meridiano $86^{\circ} 42^{\prime} \mathrm{W}$ y al oeste por el meridiano $118^{\circ} 22^{\prime} \mathrm{W}$. Esto quiere decir que estamos entre las zonas UTM 11 y 16 .

Ahora regresemos a la carta topográfica de Cananea. En el archivo de metadatos.txt viene la información siguiente:

SECCIÓN 4. Localización geográfica del conjunto de datos espaciales o producto: 4.1 Localización geográfica del conjunto de datos espaciales o producto:

4.1.1 Coordenada límite al oeste: -110.3333

4.1.2 Coordenada límite al este: -110.0000

4.1.3 Coordenada límite al sur: 30.750

4.1.4 Coordenada límite al norte: 31.000

Más adelante dice lo siguiente:

5.1.2.2 Sistema de Coordenadas de Cuadrícula:

5.1.2.2.1 Universal Transversa de Mercator

5.1.2.2.1.1 Número de zona UTM: 12

5.1.2.2.1.2 Factor de escala en el meridiano central: 0.9996

5.1.2.2.1.3 Longitud del meridiano central: -111.0

5.1.2.2.1.4 Latitud del origen de proyección: 0.0

5.1.2.2.1.5 Falso este: 500000.0

5.1.2.2.1.6 Falso norte: 0.0

Con esto era suficiente para saber que para visualizar estos datos el sistema de coordenadas que debemos de utilizar es el UTM zona $12 \mathrm{~N}$.

Otra opción es revisar alguno de los archivos .prj, que son los que va a utilizar el programa QGIS.

\section{$5 \quad$ Ejemplos de aplicaciones más avanzadas}

Para finalizar esta introducción a los Sistemas de Información Geográfica (SIG's) mencionaremos algunos ejemplos de estudios en los que los SIG's han sido parte importante. 


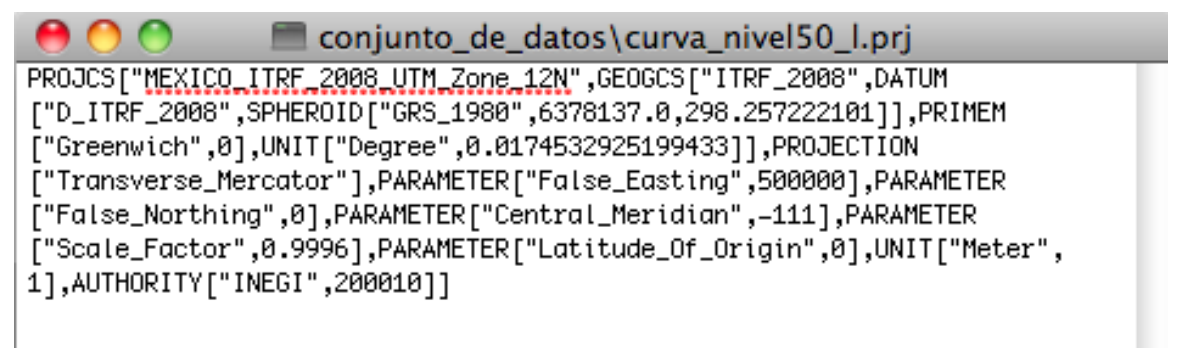

Figura 24: Contenido de un archivo .prj en el que se especifican las coordenadas UTM

1. Mapas de sismicidad. La United States Geological Survey tiene en su página [17] un registro de los sismos que ocurren en todo el mundo. En particular muestran en línea los epicentros de los sismos recientes. El Servicio Sismológico Nacional en su página [19] también muestra los epicentros de los sismos que hay en México.

2. Mapas de inundación. Parte del estudio del Plan Hídrico Integral de Tabasco incluyó modelación de inundaciones. Dicho estudio se puede consultar en la página de la Comisión Nacional del Agua (Conagua) [9]. Un estudio más breve y más reciente, aunque se concentra en una localidad de Estados Unidos, es el de Strauch et. al [6] el cual se puede consultar en la página de la USGS [17].

3. Mapas de posible afectación volcánica. El Centro Nacional de Prevención de Desastres (CENAPRED) ha desarrollado una aplicación en línea [8] para visualizar las posibles afectaciones de una eventual erupción del volcán Popocatépetl. El CENAPRED también ha publicado lineamientos que requiere para elaborción de atlas de riesgos estatales y municipales.

4. Trayectorias históricas de los huracanes. El Centro Nacional de Huracanes (de Estados Unidos) lleva un registro de las trayectorias de los huracanes que se producen en cada temporada. Se pueden consultar en línea [15].

5. Maxent. En la Universidad de Princenton han desarrollado una aplicación especial para modelar la distribución geográfica de las especies. Se llama Maxent (véase [14]). Un artículo en el cual se explica este enfoque es el de Phillips et al [4].

6. Mapas de precipitación diaria. El Servicio Meteorológico Nacional (SMN) también lleva un registro diario de la precipitación diaria. Se pueden consultar en la página del SMN [18]. También se pueden consultar las normales climatológicas por estación en formato de Google Earth.

7. Sequía. En la página del SMN [18] también se monitorea la sequía.

Más aplicaciones se encuentran referidas en las páginas de otros sistemas de información geográfica, como lo son ArcGIS [7] y GRASS [11]. Invitamos al lector a navegar en ellas. 
V. GÓMEZ

\section{Referencias}

[1] DeMers, M.N., Fundamentals of Geographic Information Systems, 498 p., John Wiley and Sons, NY (2000).

[2] Neteler, M., Mitasova, H., Open Source GIS, A GRASS GIS approach, 406 p., Springer, NY (2008).

[3] Petrasova, A., Harmon, B., Petras, V., Mitasova, H.; Tangible Modelling with Open Source GIS, 135 páginas, Springer International Publishing, 2015.

[4] Phillips, S.J., Anderson, R.P., Schapire, R.E. Maximum entropy modeling of species geographic distributions, Ecological Modelling 190:231-259,2006.

[5] Snyder, J.P., Map Projections - A working manual, 383 páginas, U.S. Geological Survey Professional Paper 1395, United States Government Printing Office, Washington, 1987.

[6] Strauch, K.R., Dietsch, B.J, and Anderson, K.J., Flood-inundation maps for a 12.5mile reach of Big Papillion Creek at Omaha, Nebraska: U.S. Geological Survey Scientific Investigations Report 2015-5152, 11 p., (2015). http://dx.doi.org/10.3133/ $\operatorname{sir} 20155152$

[7] https://www.arcgis.com/features

[8] WwW.cenapred.unam.mx

[9] www.cna.gob.mx

[10] http://www.apsalin.com/convert-geodetic-to-universal-transverse-mercator . aspx

[11] https:grass.osgeo.org

[12] www.inegi.org.mx

[13] www.beta.inegi.org.mx

[14] https://www.cs.princenton.edu/ schapire/maxent

[15] https://coast.noaa.gov/hurricanes/

[16] www.qgis.org/es/site

[17] www.usgs.gov

[18] smn.cna.gob.mx

[19] www.ssn. unam.mx 